\title{
Observing and Quantifying Ocean Flow Properties Using Drifters with Drogues at Different Depths
}

\author{
Irina I. Rypina, ${ }^{\text {a }}$ Timothy R. Getscher,${ }^{a}$ Lawrence J. Pratt, ${ }^{\mathrm{a}}$ and Baptiste Mourre ${ }^{\mathrm{b}}$ \\ ${ }^{\text {a }}$ Physical Oceanography Department, Woods Hole Oceanographic Institution, Woods Hole, Massachusetts \\ ${ }^{\mathrm{b}}$ SOCIB, Palma de Mallorca, Spain
}

(Manuscript received 25 November 2020, in final form 30 April 2021)

\begin{abstract}
This paper presents analyses of drifters with drogues at different depths-1, 10, 30, and $50 \mathrm{~m}-$ that were deployed in the Mediterranean Sea to investigate frontal subduction and upwelling. Drifter trajectories were used to estimate divergence, vorticity, vertical velocity, and finite-size Lyapunov exponents (FTLEs) and to investigate the balance of terms in the vorticity equation. The divergence and vorticity are $O(f)$ and change sign along trajectories. Vertical velocity is $O\left(1 \mathrm{~mm} \mathrm{~s}^{-1}\right)$, increases with depth, indicates predominant upwelling with isolated downwelling events, and sometimes changes sign between 1 and $50 \mathrm{~m}$. Vortex stretching is one of the significant terms, but not the only one, in the vorticity balance. Two-dimensional FTLEs are $2 \times 10^{-5} \mathrm{~s}^{-1}$ after 1 day, 2 times as large as in a 400-m-resolution numerical model. Three-dimensional FTLEs are 50\% larger than 2D FTLEs and are dominated by the vertical shear of horizontal velocity. Bootstrapping suggests uncertainty levels of $\sim 10 \%$ of the time-mean absolute values for divergence and vorticity. Analysis of simulated drifters in a model suggests that drifter-based estimates of divergence and vorticity are close to the Eulerian model estimates, except when drifters get aligned into long filaments. Drifter-based vertical velocity is close to the Eulerian model estimates at $1 \mathrm{~m}$ but differs at deeper depths. The errors in the vertical velocity are largely due to the lateral separation between drifters at different depths and are partially due to only measuring at four depths. Overall, this paper demonstrates how drifters, heretofore restricted to $2 \mathrm{D}$ near-surface observations, can be used to learn about 3D flow properties throughout the upper layer of the water column.
\end{abstract}

SIGNIFICANCE STATEMENT: Drifting buoys, or drifters, are one of the oldest instruments in physical oceanography and have been used to study near-surface horizontal currents throughout the World Ocean. However, in flows with large vertical velocity or vertical shear, currents and transport properties change with depth, so conventional near-surface drifters are of limited use. This paper shows that a simultaneous release of drifters with drogues at different depths allows observing and quantifying both horizontal and vertical structure of the flow. Specifically, we present analyses of drifters with drogues at $1,10,30$, and $50 \mathrm{~m}$, which were deployed in the Mediterranean Sea in 2018. Drifter data are used to quantify such important flow properties as divergence, vorticity, vertical velocity, and finite-time Lyapunov exponents and to investigate the vorticity equation balance throughout the top $50 \mathrm{~m}$ of the water column.

KEYWORDS: Convergence/divergence; Fronts; Nonlinear dynamics; Small scale processes; Trajectories; Upwelling/downwelling; Vertical motion

\section{Introduction}

Vertical velocities in mesoscale and submesoscale flows are typically orders of magnitude smaller than horizontal velocities. Thus, the observation and quantification of upwelling and subduction is challenging, and many questions remain about the three-dimensional processes responsible for the transport and exchange of physical and biogeochemical properties between the ocean surface and the rest of the water column.

Frontal regions, where heavier water can slide underneath lighter water along the steeply sloping isopycnals, are generally characterized by enhanced vertical velocities relative to other regions of the ocean. Although neither the magnitude nor details of the resulting 3D transport associated with such frontal subduction are fully understood, frontal zones present a promising natural laboratory where subduction and upwelling can be observed and measured.

Corresponding author: Irina I. Rypina, irypina@whoi.edu
The Alboran Sea-the western part of the Mediterranean Sea-is a confluence region of two different water masses with different water densities. Here, the fresher Atlantic water, which enters through the Strait of Gibraltar, meets the saltier Mediterranean water, creating strong and persistent fronts. One such front-the Almeria-Oran front that traverses the Alboran diagonally from Almeria to Oran, just as its name suggests-was the target of a spring 2018 field experiment carried out as part of the Coherent Lagrangian Pathways from the Surface Ocean to Interior (CALYPSO) project (https://calypsodri.whoi.edu/).

We deployed drifters with drogues at depths of $1,10,30$, and $50 \mathrm{~m}$ in the vicinity of the Almeria-Oran front. Our multilayer drifter release aimed to investigate the feasibility of observing and quantifying subduction and upwelling processes through their effect on the horizontal motion of drifters at different depths. Drifters equipped with GPS trackers were released in a tight multilayer array near a strong front. Details about the drifter deployment and the concurrent hydrological observations will be given in section 2 . 


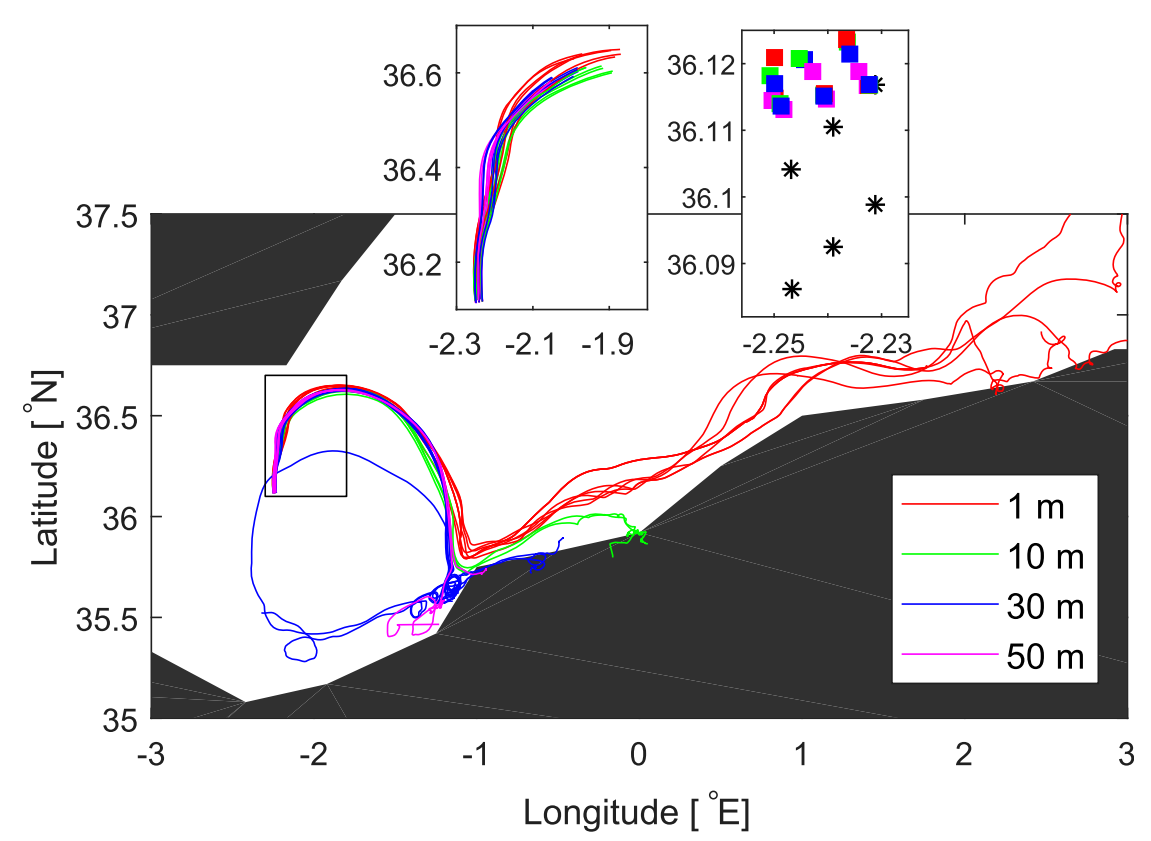

FIG. 1. Trajectories of the drifters with drogues at different depths, color coded by the drogue depth. The black-outlined rectangle demarcates the 25-h-long trajectory segments. The solid black area is land. The left inset shows a zoom-in of the 25-h-long trajectory segments. The right inset shows deployment station locations (asterisks) and drifter locations at the time when the last drifter was released (colored squares).

In this paper we present analyses from roughly the first day of drifter data, when the drifters in each layer still formed a tight cluster and when drifters in different layers were still relatively close horizontally $(<10 \mathrm{~km})$, allowing data from different layers to be stacked vertically into a three-dimensional array. Of primary interest to us are the drifter-based estimates of divergence, vorticity and vertical velocity. We also computed some of the terms in the conservation equation for vertical vorticity and divergence, and estimated finite-time Lyapunov exponents, which are often used to quantify the intensity of stirring. These analyses are presented in section 3 using methods described in section 2. Later in section 3, we use a realistic ocean circulation model to compare the Lagrangian drifter-based estimates (based on simulated drifter trajectories) for the variables of interest with the Eulerian model estimates, explore the reliability of our Lagrangian estimates, and investigate the sources of error and the underlying mechanisms responsible for the mismatch between the drifter-based Lagrangian and the Eulerian values. In section 4, we discuss and interpret our results and put them in context with prior studies.

\section{Data and methods}

\section{a. Multilayer drifter deployment}

On 29 May 2018 we deployed a multilayer drifter array of 24 drifters, with 6 drifters drogued at depths of 1, 10, 30 and $50 \mathrm{~m}$. The $1-\mathrm{m}$ drifters were of the standard Coastal Ocean Dynamics Experiment (CODE) design originally developed by Dr. R. Davis of the Scripps Institution of Oceanography; drifters of the same design were used by Rypina et al. (2016, 2014) and Chen et al. (2014) and are used by the U.S. Coast Guard for their search and rescue operations. Deeper drifters were of the holey-sock design, similar to the standard Surface Velocity Program (SVP) drifters (https://www.aoml.noaa.gov/phod/gdp/) but with a custom-length cable between the surface buoy and the subsurface holey-sock drogue. Drifters were built locally at Woods Hole Oceanographic Institution (WHOI), and the only minor operational detail worth mentioning is that we coiled the wire using pegboards to avoid entanglement during deployment. Drifters were equipped with a SPOT LLC Trace GPS tracker, which transmitted their geographical position at 5-min intervals for approximately 10 days [the same trackers were used in Consortium for Advanced Research on Transport of Hydrocarbon in the Environment (CARTHE) drifters; see D'Asaro et al. 2020]. Here we will use roughly the first day of drifter data (Fig. 1).

The drifters were released at a front near the periphery of the Eastern Alboran Gyre, about 1 day of drift upstream of the Almeria-Oran front. Targeting the Almeria-Oran front itself proved challenging because of the strong downstream velocities (reaching $1.5 \mathrm{~m} \mathrm{~s}^{-1}$ ) rapidly advecting drifters (and other instruments) into Algerian waters, where the vessel did not have permission to operate. The exact location of our target front was identified during the cruise in real time on the basis of available seawater density data from contemporaneous glider measurements and ship-based undulating CTD (u-CTD) tows and ADCP velocity measurements. During the drifter release, four drifters with drogues at 1, 10,30, and $50 \mathrm{~m}$ were deployed nearly simultaneously at each of the six stations, arranged in two rows of three stations, with neighboring stations $1-2 \mathrm{~km}$ 
apart from each other (gray asterisks in the inset of Fig. 1). The deployment took less than $2 \mathrm{~h}$. The estimation of the velocity gradients that are needed for computing divergence and vorticity works best when drifters are close together and form nearly equidistant rather than elongated polygons. The latter tend to form spontaneously in the ocean due to advection of drifters by oceanic currents, especially in regions with strong horizontal strain or shear, such as near a front associated with a strong jet. To compensate for the unwanted advection of drifters during the deployment, a "dynamic deployment" strategy was employed, where ADCP velocity data and modelpredicted current speeds were used to project drifter deployment locations forward in time to the end of deployment, so that the desired drifter configuration is achieved at the end of deployment. This dynamic deployment strategy worked well and produced a tight approximately $2-\mathrm{km}$ by $2-\mathrm{km}$ cluster of drifters by the time when the last, 24th, drifter was released (Fig. 1).

\section{b. Ancillary datasets}

To interpret and put into context the drifter-based observations, we have used two additional ancillary datasets-the satellite-based estimates of sea surface height ( $\mathrm{SSH}$ ) and the corresponding geostrophic velocities, and the in situ CTD and ADCP observations along several sections occupied during the same cruise. The SSH maps and the associated geostrophic velocities correspond to the level-4 gridded maps product publicly available from the Copernicus website (https://resources.marine.copernicus.eu/?option $=$ com_csw\& view $=$ details \& product_id=SEALEVEL_MED_PHY_ CLIMATE_L4_REP_OBSERVATIONS_008_056). The CTD data came from the u-CTD module that was towed behind the ship during the cruise, and the ADCP data came from the shipmounted downward-looking ADCP instrument.

\section{c. Estimation of velocity gradient, divergence, and vorticity}

To obtain estimates of divergence and vorticity from drifter data, we first estimated horizontal velocities by finite-differencing drifter positions and then applying the linear least squares (LLS) method of Molinari and Kirwan (1975) to obtain horizontal velocity gradients from drifter velocities. This method is sensitive to the number of drifters, distance between drifters, and geometry of the polygon spanned by the drifters. It works best for tight equidistant clusters containing many drifters, and the skill of the method deteriorates with decreasing number of drifters and increasing area and aspect ratio (i.e., ratio of the longest to shortest dimension) of the polygon spanned by the drifters. This method has recently been tested by S. Essink as part of his Ph.D. thesis (Essink 2019) in the context of a highresolution ( $500 \mathrm{~m}$ in the horizontal plane) numerical model of a baroclinically unstable front and was shown to give reasonable agreement $\left(R^{2}>0.6\right)$ with the Eulerian model vorticity for clusters of six simulated drifters, drifter separation of about $3 \mathrm{~km}$, and polygon aspect ratios $<10$. With these results in mind, we restricted our analyses of real multilayer drifters to the time period during which the polygon spanned by the six drifters in each layer is $<10 \mathrm{~km}^{2}$ and the aspect ratio is $<10$. In this paper, following Essink (2019), we characterized the geometric configuration of the drifters using two parameters- the area of the polygon spanned by the drifters and the aspect ratio of the polygon. However, other metrics for quantifying the shape of the drifter polygon are also possible. For example, Berta et al. (2016) used two alternative metrics for characterizing the geometry of the drifter triads and investigating the triad evolution - the ratio of the area to the perimeter of the triangle and a shape parameter, which depends on the largest internal angle and the ratio of the smallest to intermediate triangle sides. Since the drifter array in the present study is different from the triad configuration, it is not straightforward to directly relate the two sets of metrics.

An alternative, independent way of estimating horizontal divergence is from the change in area of the drifter polygon,

$$
\operatorname{div}(u)=\frac{1}{A} \frac{d A}{d t}
$$

To understand the physical meaning of the equality in Eq. (1), imagine a small rectangular water parcel with an area $A=$ $\Delta x \Delta y$. In a nonuniform flow, the parcel will change its area because different sides of the rectangle will move with different speed. Then the change in area can be written as

$$
\begin{aligned}
\frac{d A}{d t} & =\Delta x \frac{d(\Delta y)}{d t}+\Delta y \frac{d(\Delta x)}{d t} \\
& =\Delta x[v(y+\Delta y)-v(y)]+\Delta y[u(x+\Delta x)-u(x)] \\
& =A\left\{\frac{[v(y+\Delta y)-v(y)]}{\Delta y}+\frac{[u(x+\Delta x)-u(x)]}{\Delta x}\right\}
\end{aligned}
$$

Dividing by $A$ and taking the limit as both $\Delta x$ and $\Delta y \rightarrow 0$ gives Eq. (1). The area method is a standard technique and is described in many classic oceanographic and meteorological texts, such as Holton and Hakim (2013), Dutton (2002), or Pedlosky (1987).

Both the area-averaged vertical vorticity and the areaaveraged divergence within the drifter polygon can be estimated using the Green's theorem by evaluating the circulation around the drifter polygon and the total flux out of the drifter polygon,

$$
\begin{aligned}
\iint_{A} \operatorname{curl}_{2 \mathrm{D}}(\mathbf{u}) d a & =\oint \mathbf{u} \cdot d \mathbf{s} \quad \text { and } \\
\iint_{A} \operatorname{div}(\mathbf{u}) d a & =\oint \mathbf{u} \cdot \hat{\mathbf{n}} d s,
\end{aligned}
$$

respectively. Here $A$ is the area of the drifter polygon, $d \mathbf{s}$ and $\hat{\mathbf{n}} d s$ are unit vectors that are, respectively, tangential and normal to the perimeter of the polygon, and the integrals in the right-hand sides are taken all the way around the polygon.

\section{d. Estimation of vertical velocity}

Using the continuity equation, we can write $d u / d x+d v / d y=$ $-d w / d z$. Integrating this equation from the surface $(z=0)$ down to some depth $z(z<0)$ gives

$$
w(z)=w(0)-\int_{z}^{0} d z\left(\frac{d u}{d x}+\frac{d v}{d y}\right) .
$$


We will assume that $w(0)=0$. Vertical motions at the sea surface are mainly due to surface gravity waves and tides. Gravity waves have short temporal and spatial scales, so they are unlikely to be important on the scales of $O(1 \mathrm{~km})$ and $O(10 \mathrm{~min})$ that are resolved by the drifters. The strongest tidal component in the Alboran Sea is M2, which has an amplitude of about $20 \mathrm{~cm}$ in the geographical region of the Eastern Alboran gyre (Albérola et al. 1995). The resulting tidally induced vertical velocities are approximately $10^{-5} \mathrm{~m} \mathrm{~s}^{-1}$, which is one to two orders of magnitude smaller than the estimated drifter-based vertical velocities at and below $10 \mathrm{~m}$. The assumption of zero vertical velocity at the surface is also consistent with the numerical model simulations, for which the vertical velocities at the surface associated with the temporal variations of the sea level are two orders of magnitude smaller than the vertical velocities found at and below $10 \mathrm{~m}$. Using summation instead of integration allows for estimation of vertical velocity at each of the drifter depths as

$$
\begin{aligned}
& w\left(z_{n}\right)=-\sum_{i=1}^{n} \frac{1}{2}\left[\left(\frac{d u_{i}}{d x}+\frac{d v_{i}}{d y}\right)+\left(\frac{d u_{i-1}}{d x}+\frac{d v_{i-1}}{d y}\right)\right] \Delta z_{i}, \quad \text { for } \\
& n=1, \ldots, 4
\end{aligned}
$$

where the subscript 0 corresponds to the ocean surface; $n=$ $1, \ldots, 4$ is the level number at which drifter data are available, with $n$ increasing downward; and $\Delta z_{i}$ is the vertical distance between the neighboring drifter levels (i.e., $\Delta z_{i}=$ $z_{i}-z_{i-1}=-1,-9,-20$, and $-20 \mathrm{~m}$ in our case); divergence at the surface is assumed to be equal to that for the level immediately below (i.e., $1 \mathrm{~m}$ in our case), and summation goes over all levels above $n$. Equation (2) assumes piecewise-constant vertical profile of horizontal divergence with the algebraic mean divergence value (one-half of the sum of the values at top and bottom of each layer) throughout each layer. Assuming a piecewise-linear profile of horizontal divergence gives the same result as Eq. (2). Both assumptions work well for small $\Delta z_{i}$, that is, when the layers are thin on the vertical scale of variation of $w$ but start to fail for thicker layers.

\section{e. Estimation of the aspect ratio of the drifter polygon}

To estimate the aspect ratio $r$ of a (two dimensional) polygon spanned by the drifters in each layer, we first compute a dispersion ellipse [as in Rypina et al. $(2012,2016)$ and Kamenkovich et al. (2015)] using drifter positions $x_{i}(t), y_{i}(t)$, $i=1, \ldots, N$, where $N$ is the number of drifters $(N=6$ in our case), and then take the square root of the ratio between the major and minor axes of the ellipse,

$$
r=\sqrt{D_{\tau} / D_{n}}
$$

Here

$$
\begin{gathered}
D_{\tau}=D_{x x} \cos ^{2} \theta+D_{x y} \sin 2 \theta+D_{y y} \sin ^{2} \theta \quad \text { and } \\
D_{n}=D_{x x} \sin ^{2} \theta-D_{x y} \sin 2 \theta+D_{y y} \cos ^{2} \theta
\end{gathered}
$$

are the components of the dispersion tensor in the major and minor direction,

$$
\tan 2 \theta=\frac{2 D_{x y}}{D_{x x}-D_{y y}}
$$

gives the angle $\theta$ between ellipse's major axis $D_{\tau}$ and longitudinal direction, and

$$
\begin{aligned}
& D_{x x}=\frac{1}{N} \sum_{i=1}^{N}\left(x_{i}-\bar{x}\right)^{2}, \quad D_{y y}=\frac{1}{N} \sum_{i=1}^{N}\left(y_{i}-\bar{y}\right)^{2}, \quad \text { and } \\
& D_{x y}=\frac{1}{N} \sum_{i=1}^{N}\left(x_{i}-\bar{x}\right)\left(y_{i}-\bar{y}\right)
\end{aligned}
$$

are the components of the $2 \times 2$ single-particle dispersion tensor in Cartesian coordinates, with the overbar denoting ensemble average.

In three dimensions, the components of the $3 \times 3$ dispersion tensor in Cartesian coordinates can similarly be computed as

$$
D_{i j}=\frac{1}{N} \sum_{i=1}^{N}\left(x_{i}-\overline{x_{i}}\right)\left(x_{j}-\overline{x_{j}}\right), \quad i, j=1,2,3 .
$$

The 3D dispersion matrix given in Eq. (5) can be put in a diagonal form, with the three eigenvalues of $D_{i j}$ on the diagonal, by using a coordinate system given by the three eigenvectors of $D_{i j}$. By properties of symmetric positive-semidefinite matrices, the largest diagonal element of the dispersion matrix in any reference frame is smaller or equal than its largest eigenvalue. Thus, the largest eigenvalue and its corresponding eigenvector define the direction and size of the major axis of the dispersion ellipsoid.

\section{f. Estimation of finite-time Lyapunov exponents}

The finite-time Lyapunov exponent (FTLE) is a measure of the largest exponential separation rate between a trajectory and its closest neighbors from all directions (Haller 2002, 2015). Equivalently, it is an exponential rate at which an infinitesimal circular element (or ball in 3D) of fluid stretches into an ellipse (or ellipsoid in 3D). Denoting the initial circle diameter by $d_{0}$ and the major axis of the resulting ellipse at some later time $T$ by $d$, the FTLE can be estimated as

$$
\lambda=\frac{1}{T} \log \frac{d}{d_{0}},
$$

per its definition above. To calculate FTLEs based on real drifters, we use the best-fit dispersion ellipse in 2D or ellipsoid in 3D to estimate $d$ and $d_{0}$ and then apply Eq. (6) to compute FTLE. Note, however, that because real drifters were not released in a circle, nor were they initially aligned with the most unstable direction of the flow, this method works poorly at short times but improves over longer times after drifters realign themselves in the unstable flow direction.

In numerical models, FTLEs are rarely computed using Eq. (6) because it is computationally intensive and requires releasing a large number of particles around each location where one wants to estimate FTLEs. Instead, FTLEs are most often estimated in models by releasing dense grids of regularly spaced simulated trajectories and then computing the largest eigenvalue $\sigma$ of the Cauchy-Green tensor $G$ evaluated at each particle's initial location using trajectories of its four (in 2D) or six (in 3D) closest neighbors (Haller 2002; Lekien and Ross 2010); that is, 


$$
\lambda=\frac{1}{T} \ln \sqrt{\sigma}
$$

Here, $G=\left(\Delta x_{i} / \Delta x_{o, j}\right)^{\mathrm{T}}\left(\Delta x_{i} / \Delta x_{o, j}\right)$ and $\Delta x_{i}$ and $\Delta x_{0, i}$ denote the final and initial distance in the $i$ th direction between initially nearby particles. The advantage of this algorithm is its robustness. The disadvantage is the necessity of a large set of regularly spaced trajectories started on a dense orthogonal mesh. This method is thus not applicable to an irregularly spaced cluster of six real drifters.

A modification of the above algorithm for unstructured meshes was proposed by Lekien and Ross (2010). Given the initial and final positions of $N$ particles, FTLE at the initial position of the $i$ th particle can be estimated as

$$
\lambda=\frac{1}{T} \ln \sqrt{\tilde{\sigma}}
$$

where $\tilde{\sigma}$ is the largest positive singular value of a matrix

$$
\tilde{\mathbf{M}}=\mathbf{D X}_{f}\left(\mathbf{D X}_{0}\right)^{\mathrm{T}}\left[\mathbf{D X _ { 0 }}\left(\mathbf{D} \mathbf{X}_{0}\right)^{\mathrm{T}}\right]^{-1}
$$

that minimizes $\left\|\mathbf{D} \mathbf{X}_{f}-\mathbf{M D X}_{0}\right\|$.

Here,

$$
\begin{aligned}
\mathbf{D X}_{0} & =\left(\begin{array}{rlr}
x_{1}^{0}-x_{i}^{0} & \ldots & x_{N-1}^{0}-x_{i}^{0} \\
y_{1}^{0}-y_{i}^{0} & \ldots & y_{N-1}^{0}-y_{i}^{0} \\
z_{1}^{0}-z_{i}^{0} & \ldots & z_{N-1}^{0}-z_{i}^{0}
\end{array}\right) \quad \text { and } \\
\mathbf{D X}_{f} & =\left(\begin{array}{rlr}
x_{1}^{f}-x_{i}^{f} & \ldots & x_{N-1}^{f}-x_{i}^{f} \\
y_{1}^{f}-y_{i}^{f} & \ldots & y_{N-1}^{f}-y_{i}^{f} \\
z_{1}^{f}-z_{i}^{f} & \ldots & z_{N-1}^{f}-z_{i}^{f}
\end{array}\right)
\end{aligned}
$$

velocity (i.e., $\Delta z / \Delta x_{0}=\Delta z / \Delta y_{0}=0, \Delta z / \Delta z_{0}=1$ in the CauchyGreen tensor) - and studied whether FTLE 3d2d $_{2}$ can be used to approximate the full 3D FTLEs in two idealized flows. The reduced formulation worked well for the quadrupole model flow but not the ABC flow. Because drifters are confined to stay at the same depth, the estimation of the full 3D FTLEs is not possible using drifter data, and our drifter-based 3D FTLEs resulting from fitting the ellipsoid to the drifter data and then applying Eq. (6) only allows estimating FTLE $_{3 \mathrm{~d} 2 \mathrm{~d} \text {. For sim- }}$ plicity, we will still refer to such estimates as 3D FTLEs, to distinguish them from the 2D FTLE estimates at a fixed depth.

A simple geometrical argument can be used to estimate the contribution to 3D FTLEs from the vertical shear of horizontal velocity $\lambda_{\text {shear. In the velocity field with only vertical and no }}$ horizontal shear and no vertical velocity $(w=0)$, a small ball of fluid with initial diameter $d_{0}$ will tilt and elongate in the vertical plane (denoted $x-z$ ), keeping its horizontal extent in the perpendicular direction $y$ unchanged. If we take the vertical $x-z$ cross section of the resulting tilted ellipsoid, the major axis $d$ of the tilted ellipse in the $x-z$ plane at time $T$ is

$$
d(T)=\sqrt{d_{x}^{2}+d_{z}^{2}}
$$

where $d_{x}$ and $d_{z}$ denote the horizontal and vertical components of $d$. Because $w=0, d_{z}=d_{z 0}=$ const $(=49 \mathrm{~m}$ in our case). Assuming that the initial diameter of the ball is small, i.e., $d_{0} \ll d_{x}$, the horizontal component can be approximated by $d_{x}(t) \cong \int \Delta v(t) d t$, yielding

$$
\lambda_{\text {shear }}=\frac{1}{T} \log \frac{\sqrt{d_{x}^{2}+d_{z}^{2}}}{d_{0}}=\frac{1}{T} \log \frac{\sqrt{\left[\int \Delta v(t) d t\right]^{2}+d_{z_{0}}^{2}}}{d_{0}} .
$$

Since a particle trajectory is an integral quantity of velocity, the total FTLE is not a sum of its components coming from the contributions from the horizontal and vertical velocity and horizontal and vertical shear. Nevertheless, comparing $\lambda_{\text {shear }}$ with full $\lambda$ is still informative for quantifying the importance of the vertical shear of horizontal velocity.

\section{g. Investigating the vertical vorticity balance following drifter trajectories}

The absolute vorticity

$$
\boldsymbol{\omega}_{a}=2 \boldsymbol{\Omega}_{\text {Earth }}+\boldsymbol{\omega}
$$

consists of two terms - vorticity due to the angular rotation of the Earth and vorticity seen in the frame of the Earth.

If we ignore friction, the evolution of the vertical, that is, $z$, component of absolute vorticity, $\omega_{a}^{(z)}$, is governed by oduced a reduced formulation of FTLEs-their FTLE which takes into account vertical shear but ignores vertical

$$
\begin{aligned}
\frac{D}{D t} \omega^{(z)} & =\left\{\omega^{(x)} \frac{\partial}{\partial x}+\omega^{(y)} \frac{\partial}{\partial y}+\left[2|\Omega| \sin \phi+\omega^{(z)}\right] \frac{\partial}{\partial z}\right\} w-\left[2|\Omega| \sin \phi+\omega^{(z)}\right]\left(\frac{\partial u}{\partial x}+\frac{\partial v}{\partial y}+\frac{\partial w}{\partial z}\right)+\frac{\frac{\partial \rho}{\partial x} \frac{\partial p}{\partial y}-\frac{\partial \rho}{\partial y} \frac{\partial p}{\partial x}}{\rho^{2}} \\
& =\left[\omega^{(x)} \frac{\partial w}{\partial x}+\omega^{(y)} \frac{\partial w}{\partial y}\right]+\left[2|\Omega| \sin \phi+\omega^{(z)}\right] \frac{\partial w}{\partial z}+\frac{\frac{\partial \rho}{\partial x} \frac{\partial p}{\partial y}-\frac{\partial \rho}{\partial y} \frac{\partial p}{\partial x}}{\rho^{2}}
\end{aligned}
$$


where $\rho$ is density and $p$ is pressure.

Of interest to us are balances between the time change of vorticity following a water parcel [the term on the lhs of Eq. (11)], tilting of horizontal vorticity into vertical vorticity by vertical velocity [first term on the rhs of Eq. (11)], stretching of the water column in vertical [second term on the rhs of Eq. (11)], and the baroclinic production of horizontal vorticity [third term on the rhs of Eq. (11)]. Some of these terms, specifically, the time change term and the stretching term, can be estimated exclusively from our drifter data, whereas the tilting and the baroclinic production terms cannot and require data from other observational platforms. Because different terms describe different physical processes, comparing and contrasting them can shed light on the physical mechanisms that govern frontal subduction and upwelling.

\section{h. Investigating the evolution of horizontal divergence following trajectories}

If we ignore friction, the evolution of divergence of the horizontal velocity, $\operatorname{div}_{H}(\mathbf{u})=\partial u / \partial x+\partial v / \partial y$, is governed by

$$
\begin{aligned}
\frac{D}{D t} \operatorname{div}_{H}(\mathbf{u})= & -\left(\frac{\partial \mathbf{u}}{\partial x} \cdot \nabla\right) u-\left(\frac{\partial \mathbf{u}}{\partial y} \cdot \nabla\right) v+\frac{1}{\rho^{2}} \nabla_{H} \rho \cdot \nabla_{H} p \\
& -\frac{1}{\rho} \nabla_{H}^{2} p+f \omega^{(z)}-\beta u .
\end{aligned}
$$

The term involving $\Delta_{H} \rho \cdot \Delta_{H} p$ can be interpreted this way: suppose one has a uniform pressure gradient in the $x$ direction (so $\partial p / \partial x=$ const) and that $\rho$ increases in the $x$ direction. Then a slightly heavier water parcel will be accelerated at a slightly smaller rate than a lighter parcel, so they will separate. It is similar for the term that involves the Laplacian of the pressure. Suppose $p_{x x}>0$. Then a parcel with a slightly larger $x$ will feel a stronger pressure gradient than a parcel with slightly smaller $x$ and the two will be accelerated at different rates. The meaning of the term $f \omega^{(z)}$ is most transparent if we consider the individual terms. For example, let us take $-f(\partial u / \partial y)$ and suppose that $\partial u / \partial y>0$. Then a parcel at a smaller $y$ will feel a Coriolis acceleration that is slightly smaller than that for a neighboring parcel at slightly greater $y$. The latter will want to turn to its right more strongly than the former, and this will lead to a change in divergence. It is similar for the term $-\beta u$, as seen by considering the case in which $u$ is uniform in $y$. Then a parcel at larger $y$ will feel a stronger Coriolis acceleration (in the $y$ direction) than a parcel at a smaller $y$, reflecting the fact that $f$ is larger at larger $y$. This leads to a change in convergence following the parcel. The vorticity and divergence equations are commonly used tools in physical oceanography and meteorology. A more complete description of these equations can be found in many classical oceanographic and meteorological texts, such as Holton and Hakim (2013), Dutton (2002), or Pedlosky (1987).

The terms that can be estimated exclusively from our drifter data are $(D / D t) \operatorname{div}_{H}(\mathbf{u}), f \omega^{(z)}$, and $-\beta u$, whereas all other terms require additional data from other observational platforms.

\section{i. Bootstrapping uncertainty analysis of divergence and vorticity}

To estimate uncertainty of the drifter-based divergence and vorticity estimates, we compute standard deviation (STD) intervals for both quantities using the bootstrapping method (Efron 1979). Specifically, we reapply the linear least squares (LLC) procedure to the six subsets composed of any five of the six drifters at each depth to generate an ensemble of six (time dependent) divergence and vorticity estimates and then compute the (time dependent) STD of this ensemble to quantify the uncertainty of our drifter-based divergence and vorticity values.

\section{j. Uncertainty in divergence and vorticity due to the GPS positioning errors}

The GPS positioning error can be approximately represented by a white noise process with the STD of $5 \mathrm{~m}\left(\sigma_{x}=5 \mathrm{~m}\right)$. This GPS location accuracy estimate is provided by the manufacturer (https://www.findmespot.com/en-ca/support/spotsatellite-gps-messenger/get-help/messaging/how-accurate-isthe-spot-satellite-gps-messenger-w) and is consistent with the additional sensitivity tests done at WHOI. Although the accuracy of the SPOT GPS Trackers may vary with geographical region due to the differences in satellite coverage, we expect similar STD values for our study area near Almeria-Oran front since the Mediterranean generally has good satellite coverage. Since errors in consecutive GPS fixes are uncorrelated, they contribute in quadrature to the velocity errors, resulting from finite differencing GPS positions in time using the centereddifference scheme, i.e., $\sigma_{u}=2^{1 / 2} \sigma_{x} /(2 \delta t)=1.18 \times 10^{-2} \mathrm{~m} \mathrm{~s}^{-1}$ for $\delta t=5 \mathrm{~min}$. To reduce noise, we apply a $10-\mathrm{min}$ ( 3 point) moving average to the velocity time series, which leads to the threefold reduction for the velocity variance, giving $\sigma_{\langle u\rangle}=$ $\sigma_{u} / 3^{1 / 2}=6.8 \times 10^{-3} \mathrm{~m} \mathrm{~s}^{-1}$. Assuming that errors in velocity are uncorrelated between neighboring drifters, which are at least $\delta x=1 \mathrm{~km}$ apart, a back-of-the-envelope upper bound on the error in the velocity gradient can be obtained as $\sigma_{\partial u / \partial x}=$ $2^{1 / 2} \sigma_{\langle u\rangle} / \delta x=9.6 \times 10^{-3} \mathrm{~s}^{-1}$. Applying the sliding 10-min moving average to the velocity gradient time series decreases the STD by $3^{1 / 2}$; that is, $\sigma_{\langle\partial u / \partial x\rangle}=5.5 \times 10^{-6} \mathrm{~s}^{-1}$. Combining two velocity derivatives into divergence and vorticity increases the STD by $2^{1 / 2}, \sigma_{\operatorname{div}(\mathbf{u})}=\sigma_{\text {vorticity }}=7.8 \times 10^{-6} \mathrm{~s}^{-1}$, and applying the $10-\mathrm{min}$ averaging to the divergence and vorticity time series reduces the errors by another $3^{1 / 2}$, giving $\sigma_{\langle\operatorname{div}(\mathbf{u})\rangle}=$ $\sigma_{\langle\text {vorticity }\rangle}=4.6 \times 10^{-6} \mathrm{~s}^{-1}$, which is an order of magnitude smaller than the local Earth Coriolis parameter $f$. Note, however, that our error estimate depends on the smoothing window (which allows to trade resolution for precision) and would have been larger if the smoothing window was shorter or not applied at all. We used the 3-point 10-min smoothing window ( 2 times the sampling interval), which coincides with the time resolution of the drifter-based velocity time series resulting from the central differencing of drifter positions. More rigorous analyses of the errors in velocity gradients, vorticity, and divergence can be found in Kirwan and Chang (1979), Ohlmann et al. (2005), Haza et al. (2014), and more recently Spydell et al. (2019). In comparison with 
Kirwan and Chang (1979), our back-of-the-envelope error estimate is less rigorous and does not account for the errors resulting from the aliasing of the undersampled motions on scales comparable to the sampling interval. Note, however, that the error estimate in Kirwan and Chang [1979, their Eq. (33)] was derived for the unbiased LLC estimator, whereas we are applying the standard LLC method. We also applied smoothing filters to velocity, velocity gradients, divergence and vorticity to suppress the errors. This smoothing is accounted for in our back-of-the-envelope error estimate but not in Kirwan and Chang (1979). We have also checked our back-of-the-envelope error estimate numerically by adding a random noise with STD of $5 \mathrm{~m}$ to the simulated drifters in the model, applying the same LLC/smoothing to the simulated drifter data as to the real data, and calculating (by definition) the variance of the resulting simulated vorticity estimates. The numerically computed vorticity variance is of the same order as our back-of-the-envelope error estimate. Note, however that our model output sampling interval $(2 \mathrm{~min})$ is only marginally smaller than the GPS sampling interval ( $5 \mathrm{~min}$ ), so this numerical procedure might still miss contributions from the aliased small scales that are present in the real ocean. Our back-of-the-envelope error estimate also does not account for the geometry of the drifter configuration and the cross-drifter error covariances; these effects have recently been investigated by Spydell et al. (2019).

\section{k. High-resolution ocean circulation model and analysis of simulated drifters}

We use a realistic ultra-high-resolution simulation of the SOCIB Western Mediterranean Operational Forecasting System (WMOP) ocean circulation modeling system implemented at SOCIB (Balearic Islands Coastal Observing and Forecasting System; Tintoré et al. 2013) to quantify uncertainties of the drifter-based estimates of divergence, vorticity and vertical velocity, and investigate the sources of errors.

The WMOP modeling system is based on a configuration of the primitive equation Regional Ocean Modeling System (ROMS) model (https://www.myroms.org) covering the western Mediterranean Sea with a spatial resolution of $2 \mathrm{~km}$ (Juza et al. 2016; Mourre et al. 2018). Boundary conditions were provided by the Mediterranean Forecasting model from the Copernicus Marine Service. To obtain the highest possible spatiotemporal resolution, two-way nesting was implemented between the $2-\mathrm{km}$ grid and a $400-\mathrm{m}$-resolution domain covering the drifter field experiment region, that is, the geographical domain where the real drifters were located during the first $25 \mathrm{~h}$ after deployment (the nested grid extends from $-3.52^{\circ}$ to $-1.02^{\circ} \mathrm{E}$ and from $35.06^{\circ}$ to $36.84^{\circ} \mathrm{N}$; see Fig. 6, described in more detail below). In the vertical direction, SOCIB WMOP model had 32 terrain-following layers, resulting in a vertical resolution varying from $2 \mathrm{~m}$ at the surface to $8 \mathrm{~m}$ at $50-\mathrm{m}$ depth in the area of the drifter deployments. The model was forced by the realistic atmospheric forcing from the Spanish Meteorological Agency HIRLAM model with a 5-km and hourly resolution. The nested simulation was initialized on 28 May 2018 from the outputs of the 2 -km-resolution WMOP reanalysis simulation that assimilated data from satellite products (temperature and along-track sea level anomalies), Argo temperature and salinity profiles, as well as from the high-frequency radar surface currents in the Ibiza Channel. The Ensemble Optimal Interpolation procedure used to assimilate the data is described in Hernandez-Lasheras and Mourre (2018). The nested simulation was then run without any further data assimilation updates. This approach allowed us to generate a simulation initialized from a state as close as possible to the observed conditions but then fully governed by the model equations. Model velocity, temperature, salinity, density, and wind stress fields within the 400-m-resolution domain were saved at 2-min temporal resolution during $48 \mathrm{~h}$ starting on 1 June 2018. Despite inevitable differences in the magnitude and exact positions of the current structures, comparisons with the data from satellites, drifters, gliders, and u-CTD sections taken during the June 2018 CALYPSO cruise showed that the model solution provided a reasonably realistic representation of the Almeria-Oran front and associated salinity gradients (Garcia-Jove et al. 2020; CALYPSO website, https://calypsodri.whoi.edu/). While horizontal velocities are explicitly solved for in the WMOP model, vertical velocities are diagnosed from the incompressibility condition at each model grid point. The Eulerian estimates of velocity gradients, as well as divergence and vorticity were estimated from finite-differencing model velocities between neighboring model grid points. Simulated drifters (Fig. 6, described in more detail below) mimicking the real multilayer drifter array were released into the ultra-high-resolution simulation at the same locations, depths, and times as real drifters and were advected by the model currents interpolated to the drifter drogue depth (i.e., 1, 10, 30 and $50 \mathrm{~m}$ ). The fourthorder variable-step Runge-Kutta integration scheme (MATLAB function "ode45") with bilinear interpolation in time and space between model grid points was used for trajectory integration. Simulated trajectories were saved at 5-min time intervals to match real drifter data. No explicit diffusion was added because the ultra-high spatiotemporal model resolution ensures that both mesoscale and dominant submesoscale motions are explicitly resolved.

Simulated drifters were analyzed in the exact same manner as real drifters, and the drifter-based divergence, vorticity, and vertical velocity estimates were compared with the Eulerian model divergence, vorticity, and vertical velocity interpolated from the model grid to the position of the center of mass of the drifter polygon. The comparison between the model Eulerian and simulated-drifter-based Lagrangian estimates was then quantified by means of the correlation coefficients. To investigate the sources of errors in the drifter-based vertical velocity estimates, two additional simulations were run. First, we used the Eulerian model divergence instead of the Lagrangian drifter-based estimated divergence following the simulated drifters at four depths (this still includes errors due to the lateral separation between drifters at four depths and due to only having four layers in vertical), and second, we used Eulerian model divergence at 4 depths following the center of mass of the drifter distribution (this eliminates errors due to the lateral separation between drifters and only includes errors due to having only four vertical layers). 
The model-based Ekman currents were estimated by using model output of wind stress and applying the Ekman layer depth formula $d(\mathrm{~m})=3.2 \mathrm{~s} \times U\left(\mathrm{~m} \mathrm{~s}^{-1}\right) /[\sin (\phi)]^{1 / 2}$, where $\phi$ is latitude and $U$ is the wind speed at $10 \mathrm{~m}$. The Ekman currents at a given depth $z(\mathrm{~m})$ were then computed as

$$
\begin{aligned}
& u_{E}=\frac{\sqrt{2}}{\rho f d} e^{-(z / d)}\left[\tau_{x} \cos \left(\frac{z}{d}-\frac{\pi}{4}\right)-\tau_{y} \sin \left(\frac{z}{d}-\frac{\pi}{4}\right)\right] \text { and } \\
& v_{E}=\frac{\sqrt{2}}{\rho f d} e^{-(z / d)}\left[\tau_{x} \sin \left(\frac{z}{d}-\frac{\pi}{4}\right)+\tau_{y} \cos \left(\frac{z}{d}-\frac{\pi}{4}\right)\right],
\end{aligned}
$$

where $f=2 \Omega_{\text {Earth }} \sin (\phi)$. Since the Ekman layer depth does not adjust instantaneously to the changing wind, we applied a 2-day running average to the model wind stress and wind speed when estimating Ekman currents.

\section{Results}

\section{a. Analysis of real drifters}

Figure 1 presents a spaghetti diagram of trajectories of 24 drifters with drogues at different depths, color-coded by the drogue depth. After deployment, drifters at all depths started to move anticyclonically around the northwestern quarter of the periphery of the Eastern Alboran Gyre, reaching the northernmost location after approximately $25 \mathrm{~h}$.

Because the oceanic currents were surface-intensified, the upper layers of drifters moved at a faster speed than the lower layers. After $25 \mathrm{~h}$, this led to an $O(10 \mathrm{~km})$ separation between drifters at the top and bottom levels, and caused the misalignment (or tilting) of the initially vertically aligned multilevel drifter array (Fig. 2a).

In a $2 \mathrm{D}$ and incompressible flow, the area of any $2 \mathrm{D}$ fluid element or conservative tracer remains constant as the flow evolves. A polygon spanned by any finite number of drifters, however, is not a material contour and is not exactly conserved even in a 2D and incompressible flow. Nevertheless, it is approximately conserved when the number of drifters is sufficiently large and the area spanned by drifters is sufficiently small so that the drifter-spanned polygon at any time is close to the true material contour resulting from the forward advection of the initial drifter-spanned polygon. Thus, large changes in the area of the drifter-spanned polygon could be indicative, at least approximately, of the presence of a significant horizontal flow divergence [here the term "flow divergence" refers to both divergence or convergence and simply means $\operatorname{div}(\mathbf{u})=d u / d x+d v / d y \neq 0]$. Over the $25 \mathrm{~h}$, the area of the polygon spanned by the six drifters in each of the four levels (Fig. 2b) increased with time. The change is more pronounced for the top level of drifters, although the area growth at that depth is not monotonic. Note also that the initial area at $1 \mathrm{~m}$ is the largest among the four levels $\left(2 \mathrm{~km}^{2}\right.$ at $1 \mathrm{~m}$ vs $1 \mathrm{~km}^{2}$ at $50 \mathrm{~m}$ ).

As is typical for general oceanic flows, a compact blob of tracer or a tight cluster of drifters will stretch into a long and narrow filament due to the presence of the time-dependent horizontal shear and strain of the horizontal oceanic velocities. The drifters at each level exhibit this expected behavior, as
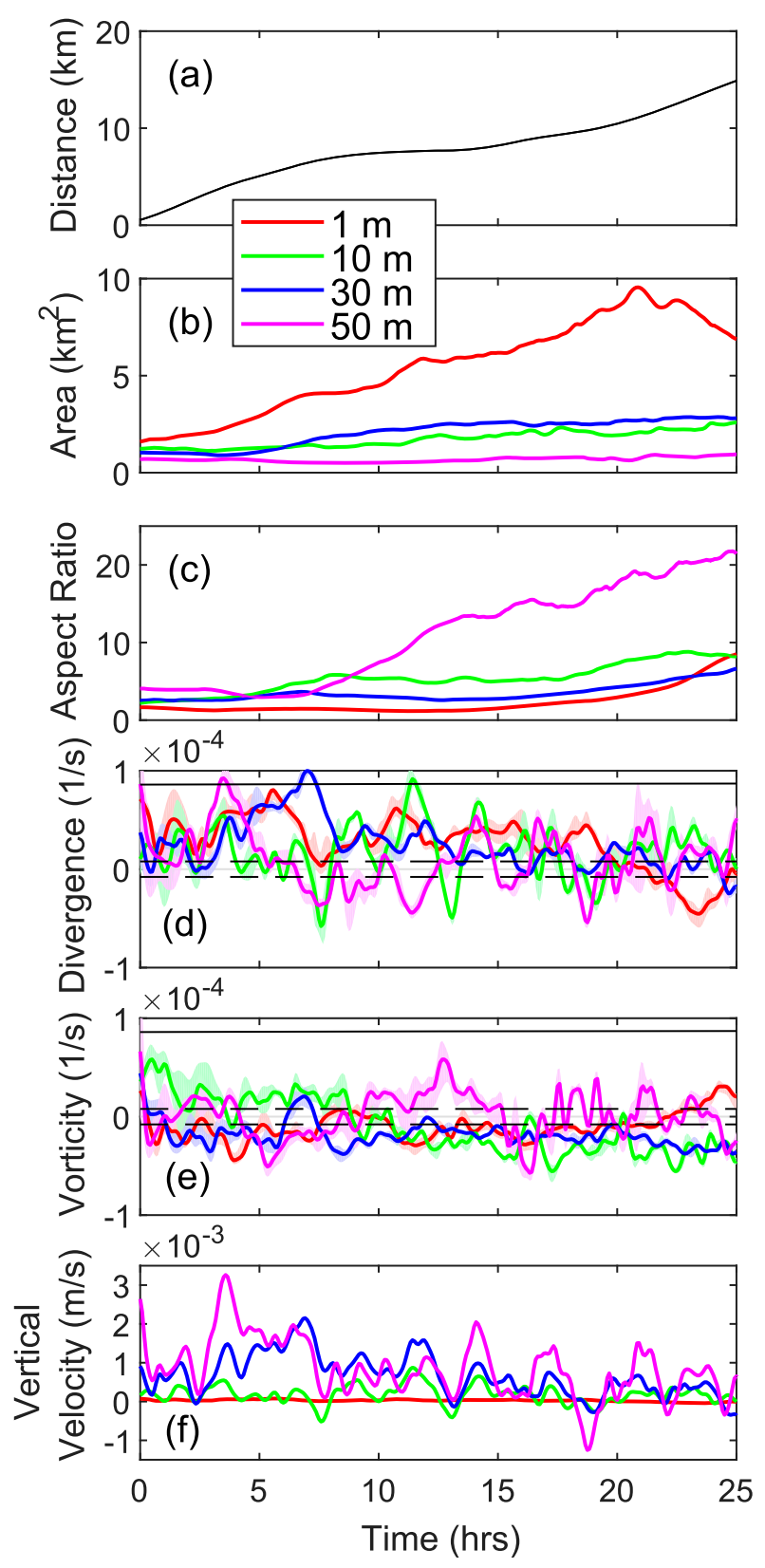

FIG. 2. Drifter-based estimates of (a) separation between the top and bottom levels of drifters (i.e., the distance between the center of mass of the drifter distribution at $1 \mathrm{~m}$ and at $50 \mathrm{~m}$ ), (b) area of the polygon spanned by the drifters, (c) aspect ratio of the polygon, (d) divergence, (e) vorticity, and (f) vertical velocity at four depths. In (d) and (e), colored shading, horizontal dashed black lines, and the solid black line show the \pm 1 -STD interval from the bootstrapping method, $\pm 1-S T D$ estimates resulting from GPS uncertainty, and the value of $f$. The color notation is as in Fig. 1.

evident from an increase in the aspect ratio of the polygon spanned by the drifters (Fig. 2c). The deepest cluster of drifters elongates more rapidly, reaching the aspect ratio of 10 after $12 \mathrm{~h}$. After $25 \mathrm{~h}$, the aspect ratios were close to or above $10 \mathrm{at}$ all four depths. 

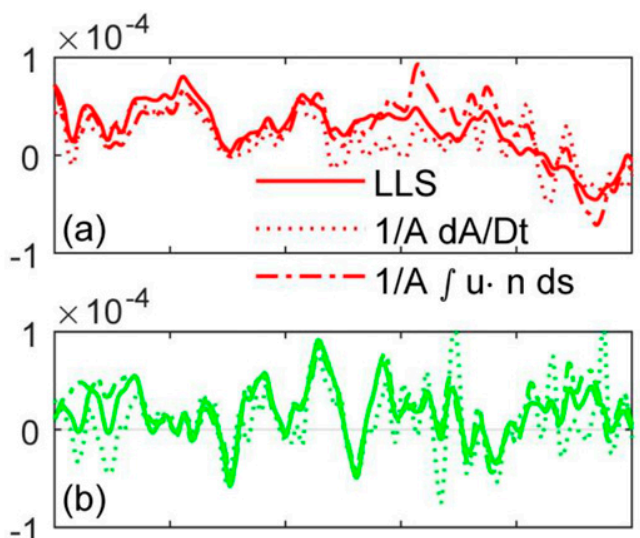

$-1$
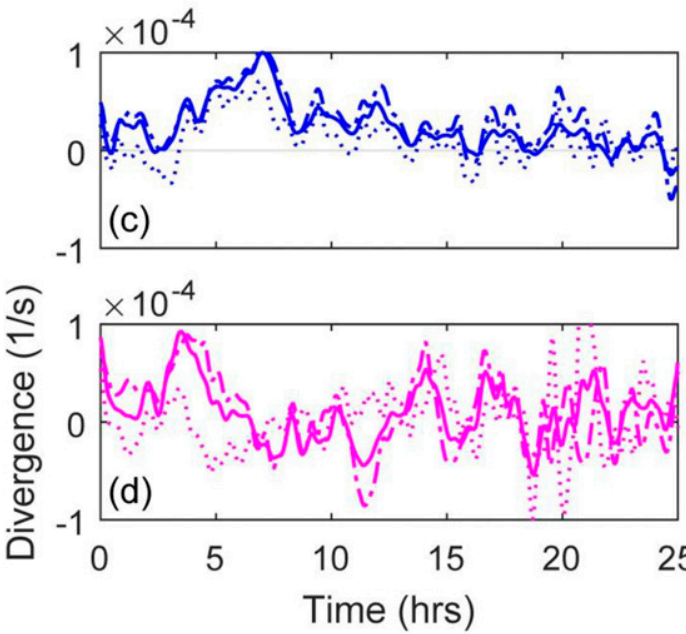
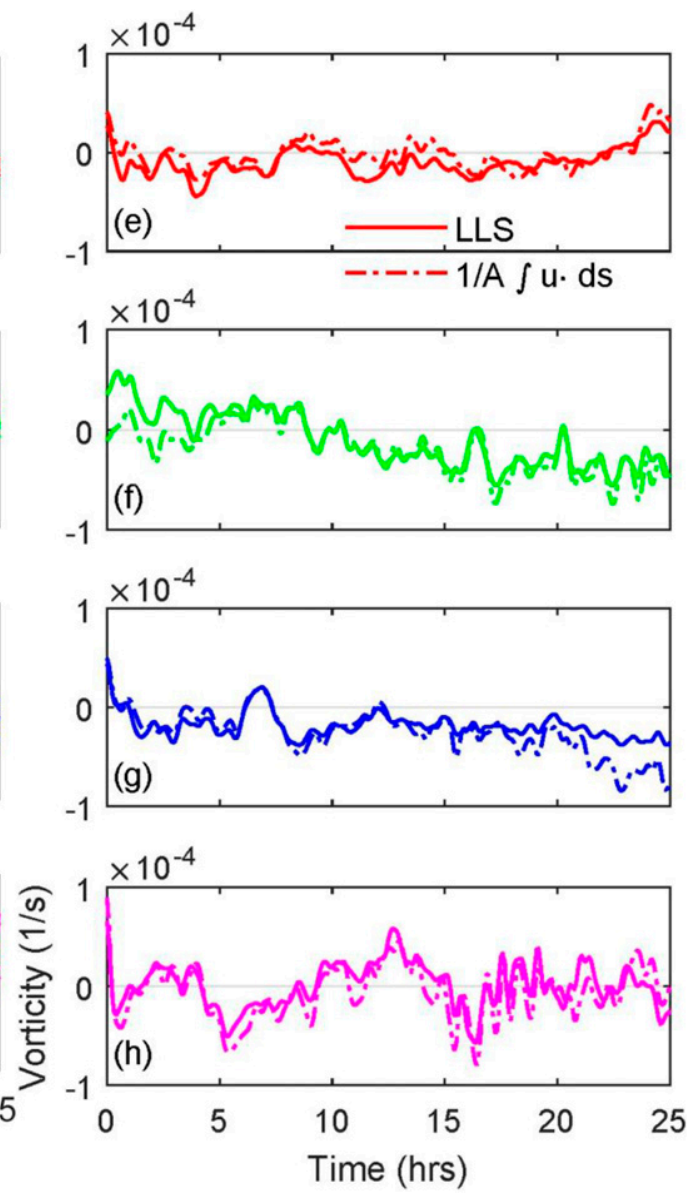

FIG. 3. Drifter-based (a)-(d) divergence and (e)-(h) vorticity estimated using different methods (as indicated by the legend) at (top) 1, (top middle) 10, (bottom middle) 30, and (bottom) $50 \mathrm{~m}$.

The drifter-based divergence and vorticity estimates obtained using the linear least squares method, along with the corresponding \pm 1 -STD interval estimated using the bootstrapping method, are shown in Figs. 2d,e. At all depths, both divergence and vorticity are $O\left(10^{-4} \mathrm{~s}^{-1}\right)$, that is, the same order of magnitude as the Earth Coriolis parameter $f$, and change sign multiple times following the drifter trajectories. Over the $25 \mathrm{~h}$, the time-averaged 1-STD uncertainty interval from bootstrapping is $O\left(10^{-5} \mathrm{~s}^{-1}\right)$, which corresponds approximately to $10 \%$ of the time-averaged absolute value for both divergence and vorticity.

The drifter-based linear least squares (LLC) divergence agrees reasonably well with the divergence estimates obtained using the area change method [Eq. (1)] and the circulation integral method [Eq. (1b)] at the top three layers (Figs. 3a-c). The correlation coefficients between the different divergence estimates over the $25 \mathrm{~h}$ are $0.7-0.9$ at 1,10 , and $30 \mathrm{~m}$. At $50 \mathrm{~m}$ (Fig. 3d), the LLC estimate correlates well with the Green's theorem estimate (correlation of 0.8 ) but does not correlate well with the area-change method (correlation of 0.33 ). The agreement is worst for the second part of the record, possibly because of the aspect ratio of the drifter-spanned polygon becoming too large ( $>10)$. This agrees with Essink (2019), who concluded based on a high-resolution numerical model that the linear least squares method of Molinari and Kirwan fails when the aspect ratio becomes too large. For vorticity, good agreement between the LLC estimate and the Green's function estimate [Eq. (1a)] is observed at all four depths, with the correlation coefficient above 0.8 (Figs. 3e-h).

The drifter-derived vertical velocity (Fig. 2f), estimated by vertically integrating drifter-based divergence, indicates predominant upwelling $(w>0)$ with only a few short isolated instances of downwelling during the first $25 \mathrm{~h}$ of drifter data. The magnitude of upwelling is generally stronger at depth than near the surface and reaches $3.3 \mathrm{~mm} \mathrm{~s}^{-1}$ at its maximum. However, during several short time instances, the vertical velocity at $50 \mathrm{~m}$ was weaker than at 10 or $30 \mathrm{~m}$. The strongest downwelling event happened near hour 19 , when vertical velocities at all depths became negative, reaching $O\left(1 \mathrm{~mm} \mathrm{~s}^{-1}\right)$ at $50 \mathrm{~m}$. Sometimes, the vertical velocity is seen to change sign between 1 and $50 \mathrm{~m}$.

Looking at the terms in the divergence equation (Fig. 4a), we estimated that both the material change in horizontal velocity divergence, $D\left[\operatorname{div}_{H}(\mathbf{u})\right] / D t$, and the $f \omega^{(z)}$ terms are $O\left(10^{-8} \mathrm{~s}^{-2}\right)$. 

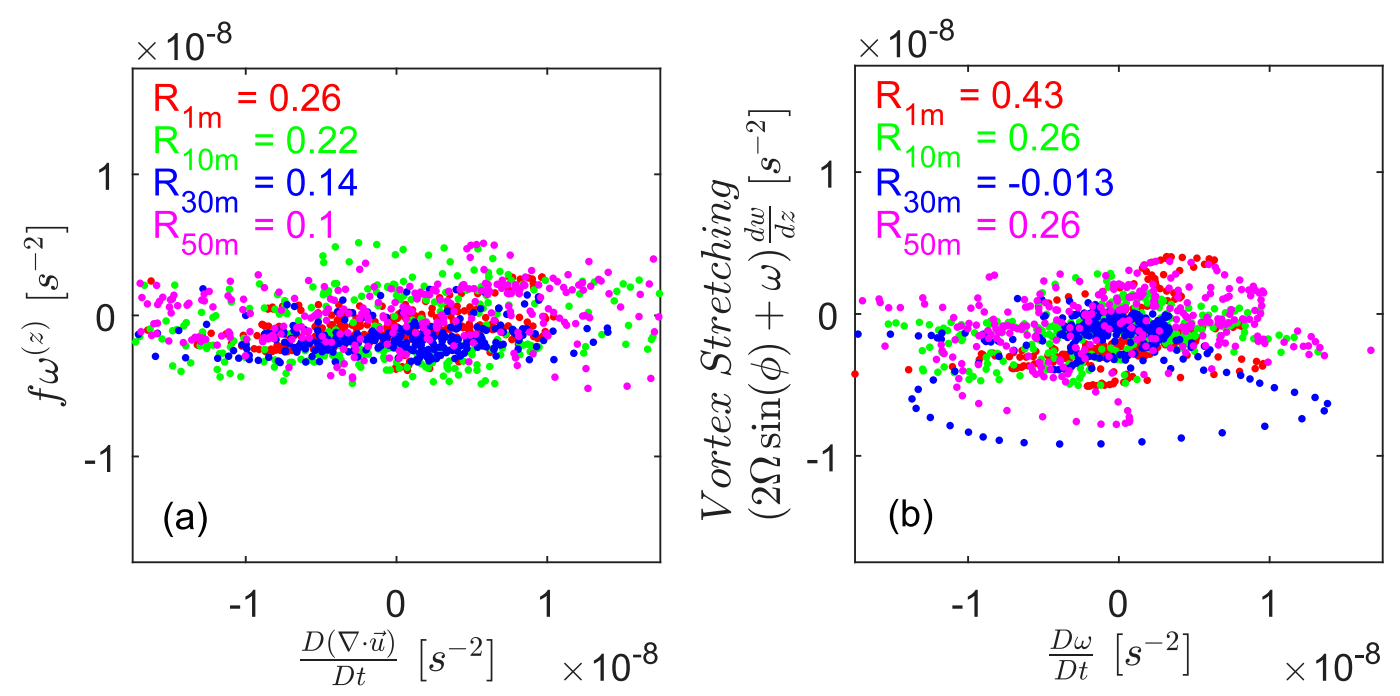

FIG. 4. The relationship between different terms in the (a) horizontal velocity divergence balance equation and (b) vertical vorticity balance equation. Correlation coefficients at different depths are shown at the top-left corner of each panel. The color notation is as in Fig. 1.

However, the two terms do not correlate well with each other, and the total range of variability for the material derivative is 4-5 times as large as for $f \omega^{(z)}$ (the distribution of dots in Fig. $4 \mathrm{a}$ is significantly elongated in the $x$ direction). The term $\beta u$ is much smaller $\left[O\left(10^{-12} \mathrm{~s}^{-2}\right)\right]$.

Comparison between the drifter-based estimate of the time rate of change of the vertical component of absolute vorticity and the vortex-stretching term in the vorticity balance is shown in Fig. 4b, again color-coded by the drifter drogue depth. The magnitudes of both terms are similar to each other and are $O\left(10^{-8}\right)$, with the range of variability being roughly 2 times as large for the rate-of-change term (from $-1.1 \times 10^{-8}$ to $1.1 \times$ $10^{-8}$ ) than for the vortex-stretching term (from $-8 \times 10^{-9}$ to $\left.5 \times 10^{-9}\right)$. At all depths, the two terms are not well correlated with each other, with the correlation coefficient between the two terms varying from -0.05 at $30 \mathrm{~m}$ to 0.45 at $1 \mathrm{~m}$.

The drifter-based FTLEs are presented in Fig. 5. The 2D FTLE estimates from the unstructured grid method indicate stronger rate of separation between drifters at upper layers relative to deeper layers. This is not surprising considering that the lateral velocities were surface-intensified. The initial segment of the 2D FTLE curve at each depth is more variable but becomes significantly smoother over the second half of the considered time interval, that is, from hour 12 until 25. The 2D FTLE values after $25 \mathrm{~h}$ are between $1.6 \times 10^{-5} \mathrm{~s}^{-1}$ at $50 \mathrm{~m}$ and $2.4 \times 10^{-5} \mathrm{~s}^{-1}$ at $1 \mathrm{~m}$. The 3D FTLEs from the best-fit ellipsoid method show a local minimum at $1 \mathrm{~h}$ followed by the local maximum at $3 \mathrm{~h}$, with a subsequent monotonic decrease in time

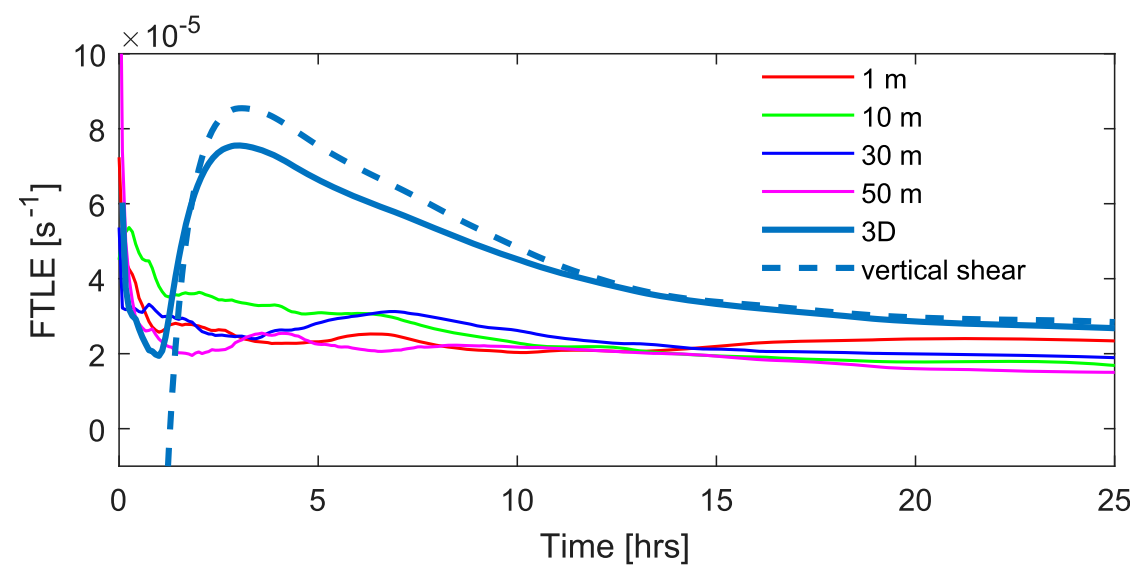

FIG. 5. Estimates of drifter-based 3D and 2D FTLEs at four depths. The 3D FTLEs were computed using the ellipsoid fit method, and the 2D FTLEs were computed using the unstructured grid method. The dashed curve shows the FTLE induced by the vertical shear of horizontal velocity. 

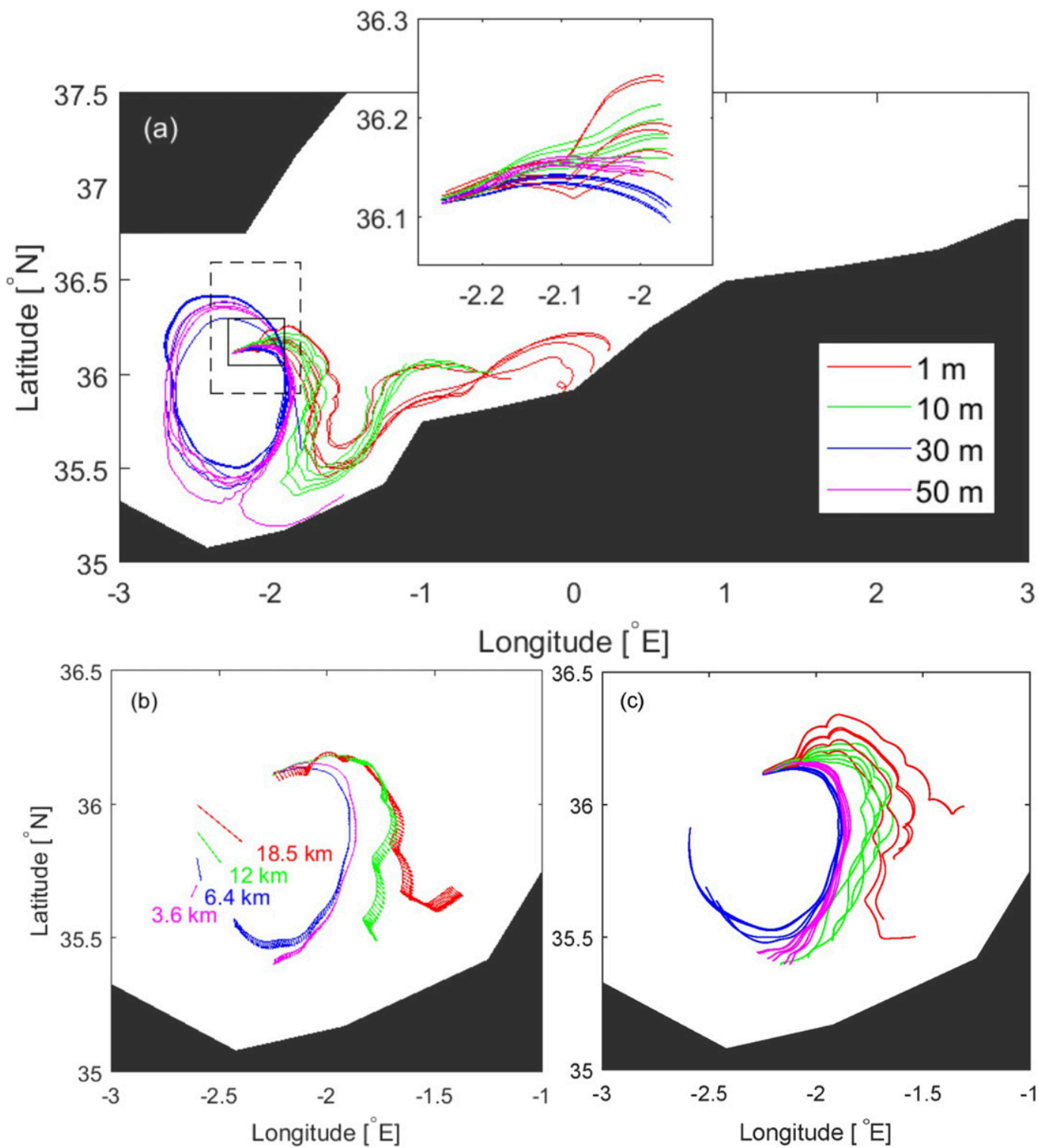

FIG. 6. (a) As in Fig. 1, but for the simulated drifter trajectories from the SOCIB WMOP model. Trajectory segments outside the 400-m-resolution model domain (dash-outlined box) and/or past $48 \mathrm{~h}$ after deployment were computed using the 2-km-resolution model velocities. The solid-outlined box demarcates the 25 -h-long trajectory segments, which are also shown in the small inset. (b) Ekman currents at the center of mass of the drifter distribution at each level every 5 min for 5 days, and the cumulative Ekman displacements after 5 days. (c) Simulated trajectories advected by the model currents with the Ekman velocity removed.

from the maximum to the value of approximately $3 \times 10^{-5} \mathrm{~s}^{-1}$ after $25 \mathrm{~h}$. The initial segment of the 3D FTLE curve, including the minimum at $1 \mathrm{~h}$, might not be trustworthy because the drifters were not initially deployed in a circle and were not initially aligned with the most unstable direction of the flow. However, starting at hour 3, the 3D FTLE curve agrees well with the semi-analytic estimate of the vertical-shear-induced drifter separation given by Eq. (9), suggesting that, first, the drifters have sufficiently realigned themselves with the unstable direction by that time so that the best-fit ellipse method becomes more reliable, and, second, that the observed drifterbased 3D FTLEs are largely dominated by the vertical shear of horizontal velocity.

\section{b. Analysis of simulated drifters}

Simulated drifters mimicking the real drifters are shown in Fig. 6a. The currents are slightly weaker in the model than in the real ocean, so the 25 -h-long segments of simulated drifters are slightly shorter than their real counterparts (black rectangle in Figs. 1 and 6a). Also, the Eastern Alboran Gyre in the 
model is smaller and slightly shifted to the southwest. Despite these differences, in qualitative agreement with real drifters, the simulated drifters at all four depths initially start to move anticyclonically around the northwestern quarter-arc of the gyre, but later split from each other, with the two upper layers moving eastward past the separation point near Oran and the two bottom layers recirculating inside the Eastern Alboran Gyre.

The splitting between the top two (1 and $10 \mathrm{~m})$ and bottom two (30 and $50 \mathrm{~m}$ ) layers is consistent with, and thus is at least partially due to, the effect of the depth-dependent wind-induced Ekman currents, shown by the colored arrows in Fig. 6 b. The Ekman spiral currents cause cumulative southeastward displacement of approximately $18.5 \mathrm{~km}$ for the $1-\mathrm{m}$ drifters and the southwestward displacement of $3.6 \mathrm{~km}$ for the $50-\mathrm{m}$ drifters after 5 days. Thus, the Ekman currents act to push the upper/lower layers of drifters out/into the Eastern Alboran Gyre. When the Ekman currents are removed from the total model velocity fields, the resulting trajectories of simulated drifters at $10 \mathrm{~m}$ are seen to recirculate inside of the Eastern Alboran Gyre instead of heading eastward past the separation region near Oran. For the 1-m trajectories, this westward shift is not strong enough to push them into the Eastern Alboran Gyre.

The simulated drifter-based displacement, area and aspect ratio of the drifter-spanned polygon, divergence, vorticity and vertical velocity are shown in Figs. $7 \mathrm{a}-\mathrm{f}$. The simulated displacement between drifters at $1 \mathrm{~m}$ and at $50 \mathrm{~m}$ after $25 \mathrm{~h}$ is $\sim 30 \%$ smaller than for the real drifters. The growth in the simulated polygon area is generally slower than that for real drifters at all depths, but especially so at $1 \mathrm{~m}$. The growth in the simulated polygon aspect ratio, on the other hand, is significantly faster than for the real drifters at $1 \mathrm{~m}$, is comparable to the real drifters at $10 \mathrm{~m}$, and is smaller at 30 and $50 \mathrm{~m}$. At $1 \mathrm{~m}$, the aspect ratio starts to grow rapidly at $15 \mathrm{~h}$ and reaches the value of 25 at $22 \mathrm{~h}$. The simulated divergence and vorticity in the model are generally smaller by a factor of 2-3 than for the real drifters, except at $1 \mathrm{~m}$, where the simulated and real values are more comparable. The model-based divergence and vorticity are also smoother. In agreement with the real drifters, the simulated divergence at all depths oscillates around zero and changes sign multiple times during the first $25 \mathrm{~h}$ after drifter deployment. Simulated vorticity at the two bottom layers $(30$ and $50 \mathrm{~m}$ ) takes both signs and oscillates around zero, just like for the real drifters. However, simulated vorticity for the top two depths ( 1 and $10 \mathrm{~m}$ ) is predominantly positive, unlike for the real drifter. In qualitative agreement with the real drifters, the vertical velocity estimates (Fig. 7f) resulting from the simulated multilayer drifters are larger at depth than near the surface, reaching $O\left(1 \mathrm{~mm} \mathrm{~s}^{-1}\right)$ at $50 \mathrm{~m}$. At all depths, vertical velocities change sign multiple times during the $25 \mathrm{~h}$, just as for the real drifters, and at some time instances, the vertical velocity has different sign at different depths. Quantitatively, the vertical velocities are smaller by about a factor of 3 in the model when compared with real drifters. Another difference is that in the model there are approximately equal amounts of upwelling and downwelling happening during the $25 \mathrm{~h}$, whereas real drifter indicated predominant upwelling.
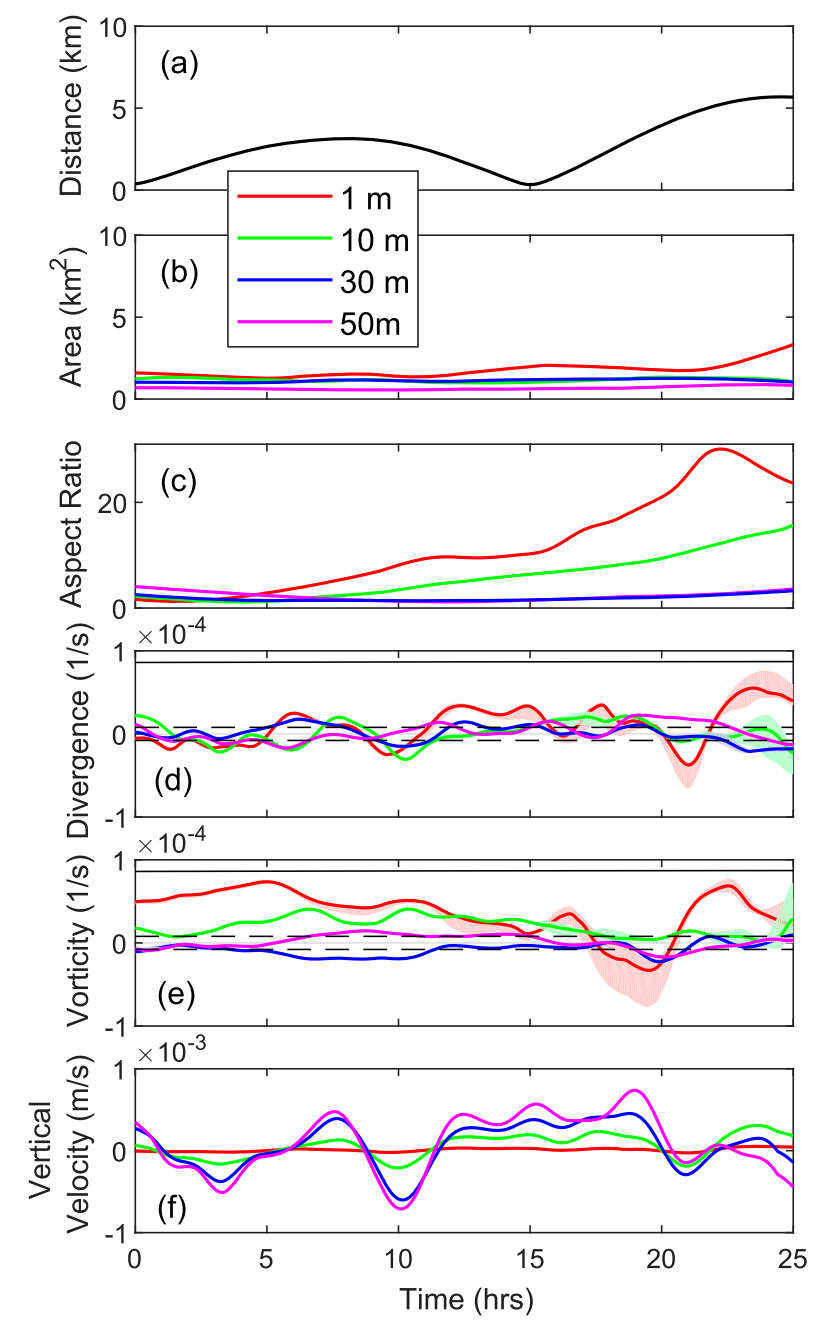

FIG. 7. As in Fig. 2, but for the simulated drifters.

In comparing the simulated-drifter-based estimates (from LLC, area-change, and Green's-theorem methods) of the divergence and vorticity with the "model truth" (Fig. 8), we see excellent agreement between all estimates (see the "divergence" and "vorticity" columns of Table 1 for the correlation coefficients), with the exception of LLC estimates at $1 \mathrm{~m}$, which disagree with truth starting at hour 15 . The poor performance of the LLC method at that time is likely due to the aspect ratio of the drifter-spanned polygon (Fig. 7c) becoming too large. Notice also that the bootstrapping STD interval for both divergence and vorticity also becomes large around the same time, indicating that the LLC estimates becomes less reliable starting at $15 \mathrm{~h}$.

For vertical velocity (Fig. 9), the correlation with the Eulerian model $w$ is relatively high at $1 \mathrm{~m}(0.71)$ but is significantly lower $(0.3-0.5)$ at the three deeper depths. The use of the Eulerian model divergence values (at the center of mass of the drifter distribution in each layer) instead of the simulated-drifter-based Lagrangian divergence estimates only leads to small improvement in the vertical velocity estimate (correlation for the three 

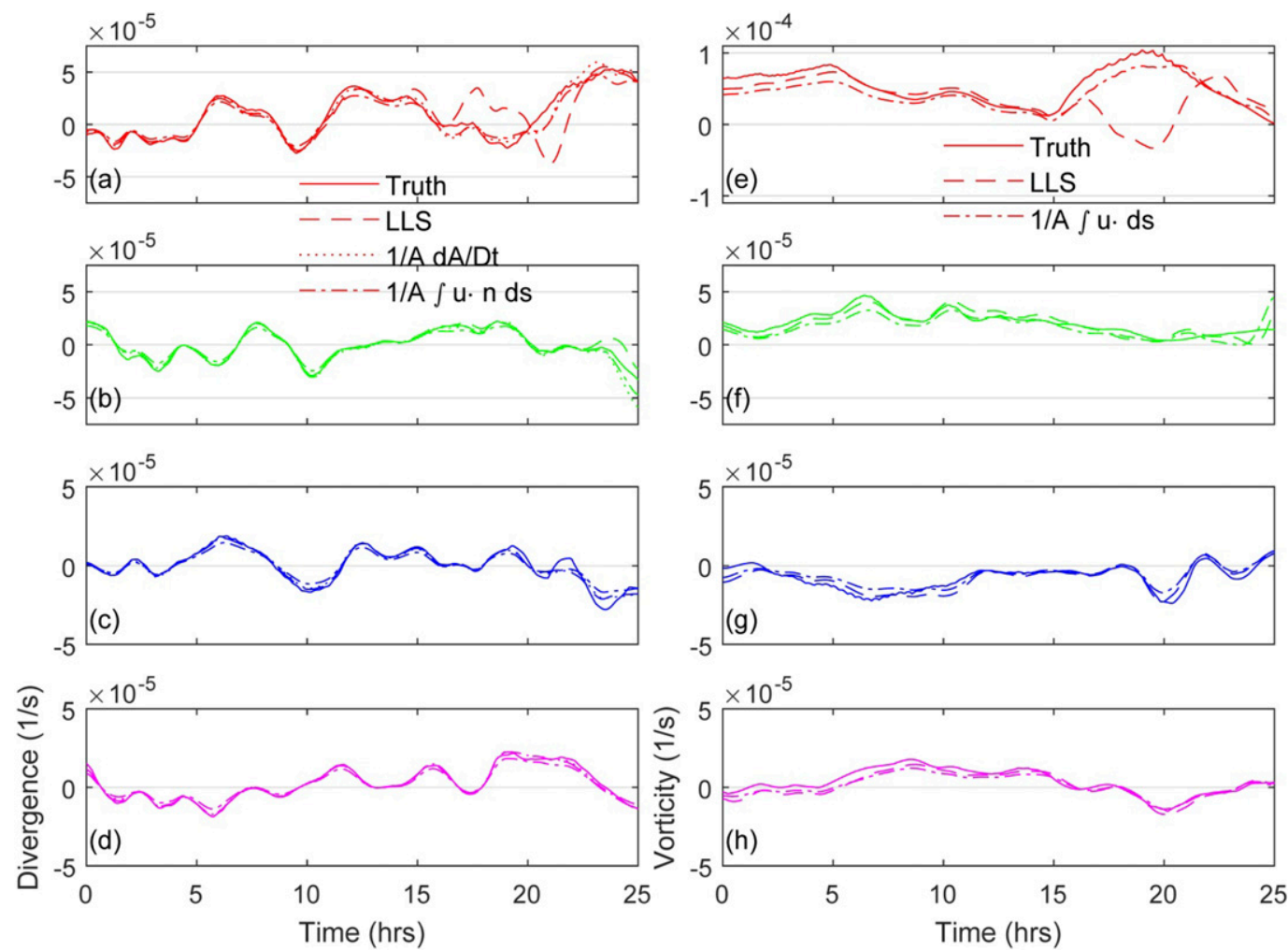

FIG. 8. Eulerian model divergence (in left panels) and vorticity (in right panels) (solid lines) and simulateddrifter-based Lagrangian (a)-(d) divergence and (e)-(h) vorticity estimated using different methods (as indicated by the legend) at (top) 1, (top middle) 10, (bottom middle) 30, and (bottom) $50 \mathrm{~m}$.

bottom layers becomes 0.5$)$. On the other hand, removing the lateral shift between four layers and using the Eulerian model divergence (still at $1,10,30$, and $50 \mathrm{~m}$ ) at the same geographical location yields significantly higher correlation with the Eulerian model vertical velocity at all depths (correlation coefficients of 0.82-0.99).

In looking at the divergence balance in the model (Fig. 10), we find that the $\beta u$ term $\left(10^{-12} \mathrm{~s}^{-2}\right)$ is again much smaller than the rate of change of horizontal velocity divergence and the $f \omega^{(z)}$ terms, which are $O\left(10^{-8}\right) \mathrm{s}^{-2}$, just as for the real drifters. The range of variability for the $(D / D t) \operatorname{div}_{H}(\mathbf{u})$ is smaller by a factor of 2-3 than for the real drifters, whereas the range of variability for $f \omega^{(z)}$ is more or less the same as for the real drifters (so the distribution of dots in Fig. 10a is less elongated in the $x$ direction than in Fig. 5a). For the vorticity balance equation, the time rate of change of vertical vorticity and the vortex-stretching terms are, on average, smaller by a factor of 2-3 relative to real drifters. In the model, the two terms generally correlate better with each other, although the correlation is still $\leq 0.6$ at all depth.

The 2D FTLE values at $25 \mathrm{~h}$ (Fig. 11) vary between $1.2 \times$ $10^{-5}$ and $2.5 \times 10^{-5} \mathrm{~s}^{-1}$, with larger values at upper depths; the 3D FTLEs are $2.75 \times 10^{-5} \mathrm{~s}^{-1}$. These FTLE values are slightly smaller but overall comparable to those obtained for the real drifters. The contribution from the vertical shear to 3D FTLEs in the model is significant, but not as strongly dominant as for

TABLE 1. For the 400-m-resolution model, correlation coefficient between the Eulerian model estimates of vorticity, divergence, and vertical velocity, and estimates obtained using different drifter-based methods. The value in parentheses corresponds to the correlation coefficient over $15 \mathrm{~h}$, instead of $25 \mathrm{~h}$ as in all other cases. Divergence, vorticity, and vertical velocity in the left three columns were obtained for the simulated multilevel drifters deployed at the same times and locations as real drifters; "4 layers, laterally separated" and "4 layers, vertically stacked" respectively refer to simulations in which Eulerian model divergence at four depths was used at the geographical locations corresponding to the center of mass of the six drifters at four depths or at the same geographical location.

\begin{tabular}{lccccr}
\hline \hline & Vorticity & Divergence & Vertical velocity & 4 layers, laterally separated & 4 layers, vertically stacked \\
\hline $1 \mathrm{~m}$ & $-0.18(0.95)$ & 0.72 & 0.71 & 0.99 & 0.99 \\
$10 \mathrm{~m}$ & 0.9 & 0.96 & 0.31 & 0.44 & 0.82 \\
$30 \mathrm{~m}$ & 0.9 & 0.96 & 0.50 & 0.48 & 0.92 \\
$50 \mathrm{~m}$ & 0.96 & 0.99 & 0.41 & 0.48 & 0.89 \\
\hline
\end{tabular}



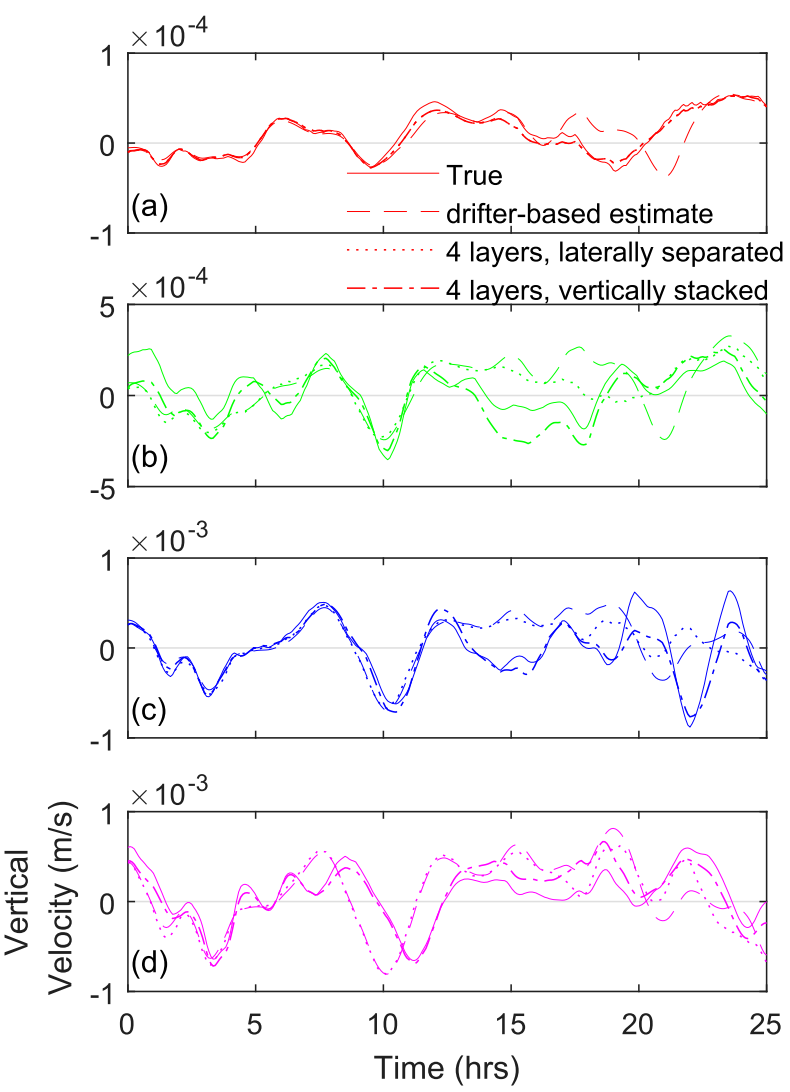

FIG. 9. At four depths [(a) 1, (b) 10, (c) 30, and (d) $50 \mathrm{~m}$ ], comparison between the Eulerian model vertical velocity (solid lines) and vertical velocity estimated using various drifter-based methods (as indicated by the legend); "4 layers, laterally separated" and "4 layers, vertically stacked" respectively refer to simulations in which Eulerian model divergence at four depths was used at the geographical locations corresponding to the center of mass of the six drifters at four depths or at the same geographical location.

the real drifters. The relatively smaller role of the vertical shear in comparison with horizontal shear in the model is consistent with, and is likely due to, the larger spread of the simulated drifters within the two layers and the smaller spread between the layers.

\section{c. Analysis of the concurrent $u-C T D$ and $A D C P$ measurements}

We now use contemporaneous data from the towed u-CTD and ship-based ADCP instruments, as well as the satellite maps of SSH and the inferred geostrophic velocities to interpret the behavior of drifters and put them in perspective with other types of measurements.

The existence of the surface-intensified jet, which is located near the periphery of the Eastern Alboran Gyre and which is characterized by the maximum in the horizontal velocity, is evident in the concurrent images of the satellite-based SSH (Fig. 12). On the western side of the Eastern Alboran Gyre, where the drifters were released, the jet is flowing northward. The associated maximum in the meridional velocity is clearly depicted in all of the ADCP sections (sections are labeled as \#1-\#6) taken both before and after the deployment of the multilevel drifters (Fig. 13, middle and right). The drifterbased horizontal velocity (indicated by the color of each circle in Fig. 13) is consistent with the ADCP measurements, and is in general agreement with the geostrophic velocities derived from SSH data. The northward velocities within the jet reach $O\left(1 \mathrm{~m} \mathrm{~s}^{-1}\right)$ near the surface and extend down to about $150 \mathrm{~m}$. U-CTD measurements (Fig. 13, left) taken along the same sections as the ADCP data, show nearly uniform density in the upper $25 \mathrm{~m}$ (i.e., mixed layer) and the sloping isopycnals between 25 and $150 \mathrm{~m}$, collocated laterally with the jet. Thus, the top two levels of drifters ( 1 and $10 \mathrm{~m}$ ) were located within the mixed layer, whereas the bottom two levels $(30$ and $50 \mathrm{~m}$ ) were below. The zonal (cross jet) extent of the frontal zone in all sections is $O(20 \mathrm{~km})$, which is significantly larger than the initial size of the drifter polygon (about $2 \mathrm{~km} \times 2 \mathrm{~km}$ ) at deployment. The structure of the water column is qualitatively similar between all sections (\#1-\#6). The density in this region is mostly controlled by salinity below the mixed layer (i.e., below $\sim 25 \mathrm{~m}$ ), but the contribution from temperature dominates above $25 \mathrm{~m}$ and is still nonnegligible at depth (Fig. 14). At 50-100-m depth, the density difference between the opposite sides of the front is about $1 \mathrm{~kg} \mathrm{~m}^{-3}$, which is about $33 \%$ smaller than the density difference of $1.5 \mathrm{~kg} \mathrm{~m}^{-3}$ associated with the stronger Almeria-Oran front located on the eastern side of the Eastern Alboran Gyre, where Mediterranean waters meet Alboran waters. As seen in section \#3, all drifters were deployed on the cyclonic side of the jet, just slightly to the west from its center. In the subsequent sections \#4-\#6, the top layers of drifters crossed the sections increasingly farther east from the lower layers. This suggests that, as the drifters were advected downstream, the top layers of drifters were moving toward the center of the jet, whereas the lower layer did not. This behavior of the upper layers of drifters is consistent with the presence of a secondary ageostrophic circulation with cross-front velocities at the surface and subduction happening on the heavier side of the front (Hoskins 1982). On the opposite, lighter side of the front, an upwelling of the lighter water mass is expected, but our drifters do not seem to reach down sufficiently deep to feel these effects. A comparison between the drifter locations where they crossed the various sections and the ADCP data at the sections hints that the drifters in the top layers may have crossed to the anticyclonic side of the jet as they moved farther north, whereas the deepest layer still remained on the cyclonic side. An indication of this behavior can also be seen in the drifter-derived vorticity time series. At the time of deployment, vorticity in all layers was positive, whereas 12-15 h later, when the drifters were crossing section \#6, the vorticity in the bottom layer was still positive, but in the shallower layers it had become negative (Fig. 15). Interestingly, the vorticity at $10 \mathrm{~m}$ stayed mostly positive for the first $10 \mathrm{~h}$, whereas vorticity at other levels did not. During hours 4-6, when the drifters were crossing section \#4, the 10and $30-\mathrm{m}$ drifters were located slightly farther east from the 1and 50-m drifters (Fig. 13), so one might intuitively expect a more negative vorticity at 10 and $30 \mathrm{~m}$ than at 1 and $50 \mathrm{~m}$. However, that is not the case in Fig. 15. Because the exact 


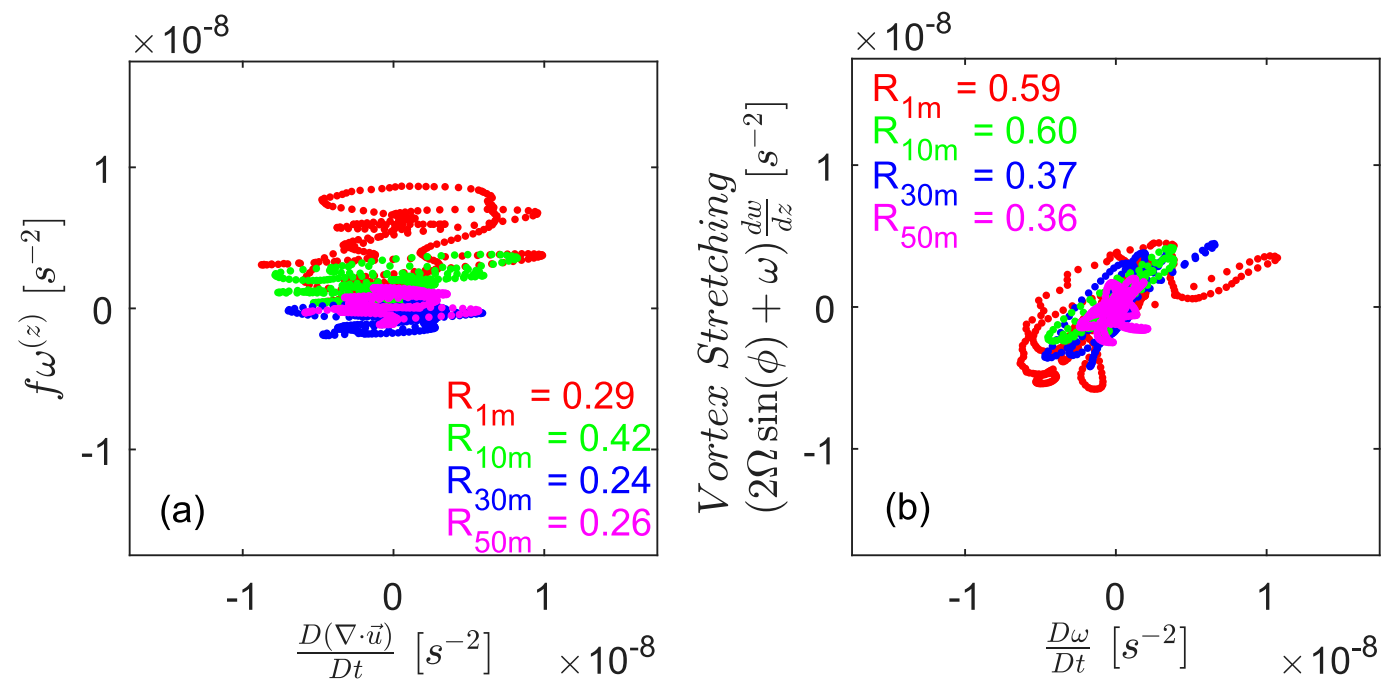

FIG. 10. As in Fig. 4, but for simulated drifters.

location of the jet core and the complicated small-scale spatiotemporal structure of the 4D vorticity field in the jet region is not well resolved by a handful of hydrological sections occupied during the cruise, it is hard to tell where exactly the drifters were with respect to the jet and the secondary circulations and it is difficult to interpret the details of the observed variability in vorticity following the drifter trajectories.

\section{Discussion}

A Lagrangian view, where one tracks individual parcels, presents a natural framework for characterizing transport and exchanges. Drifting buoys, or drifters, represent one of the oldest types of instruments in physical oceanography. Equipped with GPS positioning devices, drifters have been successfully used to study near-surface horizontal currents and transports within the top 1-15 m of the water column in many regions of the ocean. However, in flows with large vertical velocity or vertical shear, currents and transport properties change significantly with depth, so conventional near-surface drifters are of limited use. A simultaneous release of drifters with drogues at different depths can address this challenge and provide a way to observe and quantify both the horizontal and vertical structure of transport and exchanges.

To explore this idea in the real ocean, we deployed an array of 24 drifters with drogues at $1,10,30$, and $50 \mathrm{~m}$ in the Mediterranean Sea during the 2018 CALYPSO cruise, and we used the resulting drifter data to estimate divergence, vorticity and vertical velocity at four depths. Such analysis proved feasible during the first $25 \mathrm{~h}$ after the deployment. At later times, drifters in each layer stretched into long and narrow polygons with an aspect ratio of $>10$, presenting challenges for the velocity gradients estimation, and drifters in different layers separated laterally by more than $10 \mathrm{~km}$, which prevented combining divergence estimates from different depths that is required for the vertical velocity calculation.
Summarizing our findings, the drifter-derived divergence and vorticity are both $O(f)$ and change sign several times following the drifter trajectories during the first $25 \mathrm{~h}$ after deployment. Divergence and vorticity obtained using the linear least squares matrix inversion method are in general agreement with estimates obtained using alternative methods, specifically, via the area change of the drifter-spanned polygon and the Green's theorem (i.e., via the circulation integral). Bootstrapping using six sets of five drifters at each depth suggests 1-STD uncertainty interval for divergence and vorticity of approximately $10 \%$ of the time-averaged absolute value for both quantities. This uncertainty is due to both errors associated with the linear least squares matrix inversion method, as well as due to the actual spatial variability of divergence and vorticity over the spatial scale of the drifter clusters (i.e., a few kilometers). Note also that the drifter-based estimates of divergence and vorticity are representative of the means taken over the area of the drifter polygon, rather than the point values.

Drifter-inferred vertical velocity $w$ is $O\left(1 \mathrm{~mm} \mathrm{~s}^{-1}\right)$ and mostly positive (i.e., predominant upwelling), but it becomes negative during several short $[O(1 \mathrm{~h})]$ isolated downwelling events. Vertical velocity $w$ is generally larger in magnitude at depth than near surface, and it sometimes changes sign between 1 and $50 \mathrm{~m}$. The latter implies that surface divergence might be a poor indicator for locations with deep downwelling (i.e., subduction). The observed vertical velocity of up to $3 \mathrm{~mm} \mathrm{~s}^{-1}$ is approximately $2-3$ times as large as vertical velocities in an ultra-high-resolution 400-m SOCIB WMOP model of the Alboran Sea. Our drifter-based $w$ values are in general agreement with $w$ inferred from the contemporaneous 3D float trajectories and float-based ADCP measurements (E. D'Asaro and A. Scherbina 2021, personal communication; Tarry et al. 2021) that were taken during the same cruise. Note also that our deployment location was located near a strong front, where one expects enhanced vertical velocities relative to other, more quiescent regions of the ocean. The presence of 


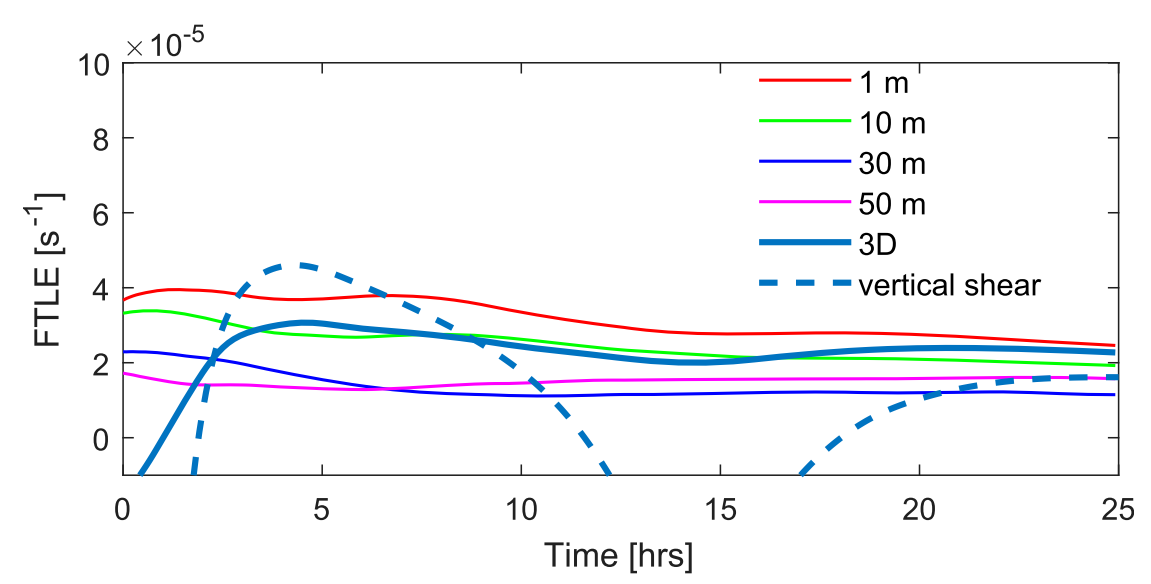

FIG. 11. As in Fig. 5, but for simulated drifters.

the density front and the associated northward jet in the proximity to the drifters was confirmed by looking at the concurrent u-CTD and ADCP sections.

From the drifter-derived divergence and vertical velocity, we estimated the time rate of change of vertical component of absolute vorticity and compared it with the vortex-stretching term from the vertical vorticity equation. At all four depths, these two terms are of the same order of magnitude but are not well correlated, suggesting that vortex stretching is one of, but not the only, significant term affecting the vertical vorticity of a water parcel. The vortex-stretching term is the only term in the vorticity equation that does not involve horizontal gradients. As such, this term is not necessarily enhanced at submesoscales relative to mesoscales, although one could also argue that the vertical velocity and its vertical shear $(d w / d z)$ can be enhanced at submesoscale, which could increase stretching. The other two terms, which we cannot estimate from drifter data, are the baroclinic production term, which converts lateral gradients of density and pressure into vertical vorticity, and the vortextilting term, which converts horizontal vorticity into vertical vorticity by acting on it via the lateral shear of vertical velocity. As both of these terms involve lateral gradients, they are expected to be larger at smaller rather than larger scales, that is, at submesoscales rather than mesoscales.

Looking at the terms in the equation governing the evolution of horizontal divergence following drifter trajectories, we estimated that the rate of change of horizontal divergence is several times as large as the $f \omega^{(z)}$ term, with the $\beta u$ term being several orders of magnitude smaller (we can only estimate these three terms using drifter data). Note that the material rates of change of divergence and vorticity in the corresponding balance equations should be evaluated following a 3D trajectory of a fluid parcel and not a 2D trajectory of drifters, as we did in this paper. Evaluating the terms following 2D drifter trajectories leads to errors due to the absence of the vertical movement of drifters and due to the not-exactly-Lagrangian nature of drifters. Because of the smallness of $w$, however, the 2D fluid parcel trajectories could be expected to stay close to the 3D fluid parcel trajectories over short time, and because of the good water-following characteristics of drifters (with a large sail and small surface buoy), the drifter trajectories could be expected to be close to the $2 \mathrm{D}$ trajectories of water parcels.

The final quantity we have investigated is the finite-size Lyapunov exponent or FTLE. Stemming from the dynamical systems theory, FTLEs have become increasingly popular over the last few decades for studying transport in geophysical flows. Maximizing ridges of FTLEs serve as proxies for hyperbolic Lagrangian Coherent Structures that delineate water parcels destined to have qualitatively different Lagrangian behavior. FTLEs are commonly computed in numerical models, but observational estimates are virtually absent. To bridge this knowledge gap, we estimated FTLEs based on our drifter

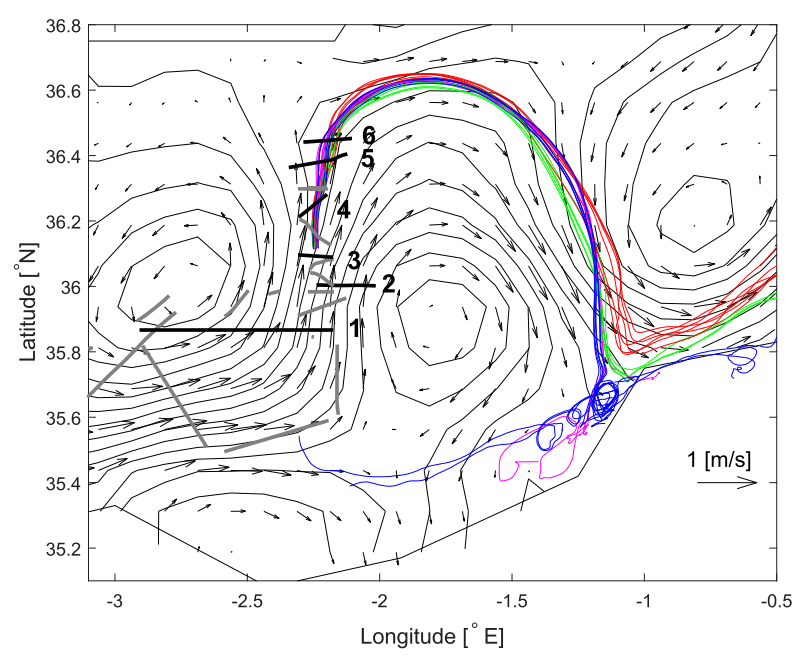

FIG. 12. Satellite-based SSH contours (thin gray) and the corresponding geostrophic currents (arrows; scale in the lower right), with the ship-based sections (thick gray and black lines) and multilevel drifter trajectories (colored curves) superimposed. The six sections that were used for our analysis are shown in black and are numbered 1-6 in the figure, with the section number indicated next to the section. The gray sections located between sections \#1 and $\# 2$, \#2 and \#3, \#3 and \#4, and \#4 and \#5 were qualitatively similar to their neighboring black sections and were omitted for brevity. 

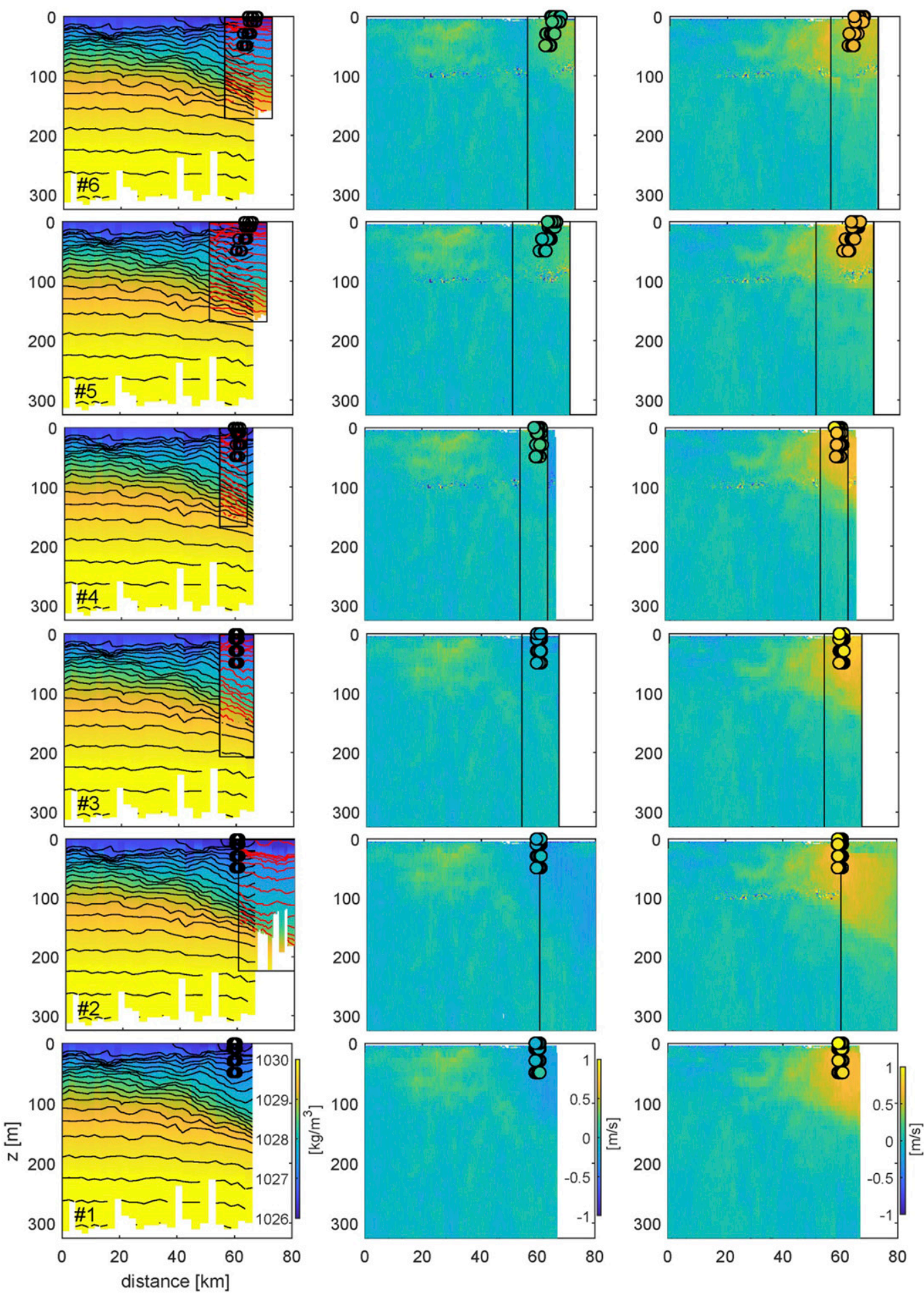

FIG. 13. For sections from (bottom) \#1 through (top) \#6, profiles of (left) density and (middle) zonal and (right) meridional velocity. All sections (black-outlined rectangles) are superimposed on section \#1 for consistency. Red curves in the left panels correspond to sections \#2-\#6 as marked. Drifters are shown by circles, color-coded in the middle and right panels by their zonal and meridional velocities, respectively. 

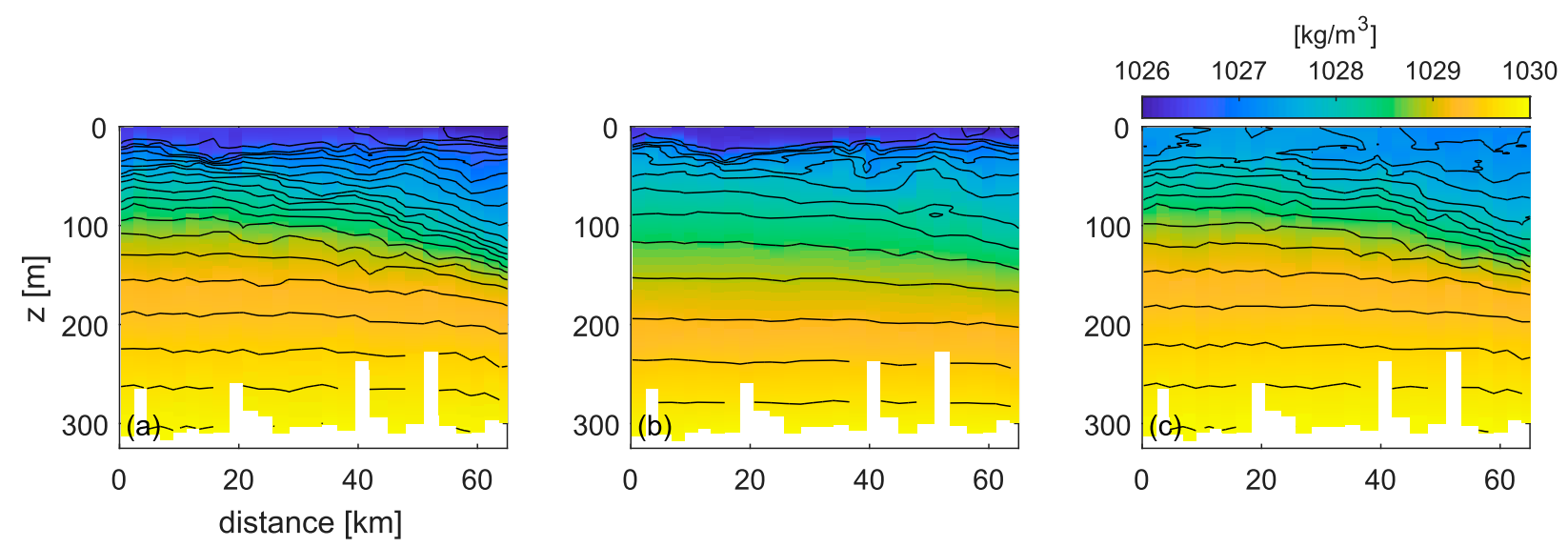

FIG. 14. Density along section \#1, computed using (a) u-CTD data for both temperature and salinity, (b) u-CTD data for temperature but fixed salinity, and (c) u-CTD data for salinity but fixed temperature.

trajectories at four depths. After $25 \mathrm{~h}$, the $2 \mathrm{D}$ drifter-based FTLEs are larger near the surface than at depth. The drifterderived FTLE values vary from $1.6 \times 10^{-5}$ to $2.4 \times 10^{-5} \mathrm{~s}^{-1}$. This is roughly 2 times as large as the corresponding $2 \mathrm{D}$ surface FTLE values from our prior studies involving highresolution $[O(2 \mathrm{~km})]$ numerical models (Rypina et al. 2010), and is comparable to 2D FTLEs from the ultra-high resolution (400-m resolution) considered in this study. This suggests that 2-km-resolution models are missing a substantial fraction of horizontal stirring imposed by the submesoscales but that $O(100 \mathrm{~m})$-resolution models represent submesoscales significantly better (although our 400-m-resolution model still underestimated divergence, vorticity, and vertical velocity relative to the real drifter-based estimates). Three-dimensional drifter-based FTLEs, which characterize the separation of our entire ensemble of 24 drifters, are $\sim 3 \times 10^{-5} \mathrm{~s}^{-1}$ after $25 \mathrm{~h}$, which again is comparable to values from the 400-m-resolution simulation. The 3D FTLEs are largely dominated by the vertical shear of horizontal velocity, as evident from the good agreement between the 3D FTLEs and their horizontal-shearinduced component.
We also used the ultra-high-resolution simulation to understand the observed splitting between the top two and bottom two layers of drifters, quantify uncertainties in the drifterbased estimates of divergence, vorticity and vertical velocity, and investigate the sources of errors. Both real and simulated multilayer drifters showed qualitative different behavior between the top two and bottom two depths. Specifically, the drifters at 30 and $50 \mathrm{~m}$ recirculated within the Eastern Alboran Gyre over several days since deployment, whereas drifters at 1 and $10 \mathrm{~m}$ were expelled from the gyre and moved eastward along the Algerian coast past the separation point near Oran. The model suggests that the wind-induced Ekman currents contributed to this splitting by pushing the top two layers of drifters eastward out of the Eastern Alboran Gyre over the first several days after deployment.

Comparing the simulated drifter-based Lagrangian estimates with the Eulerian model divergence, vorticity, and vertical velocity provides an indication of the reliability of the drifter-based values. Our analysis suggests that the errors in the drifter-derived divergence and vorticity are small, as quantified by the high $(>0.9)$ correlation coefficient between Lagrangian

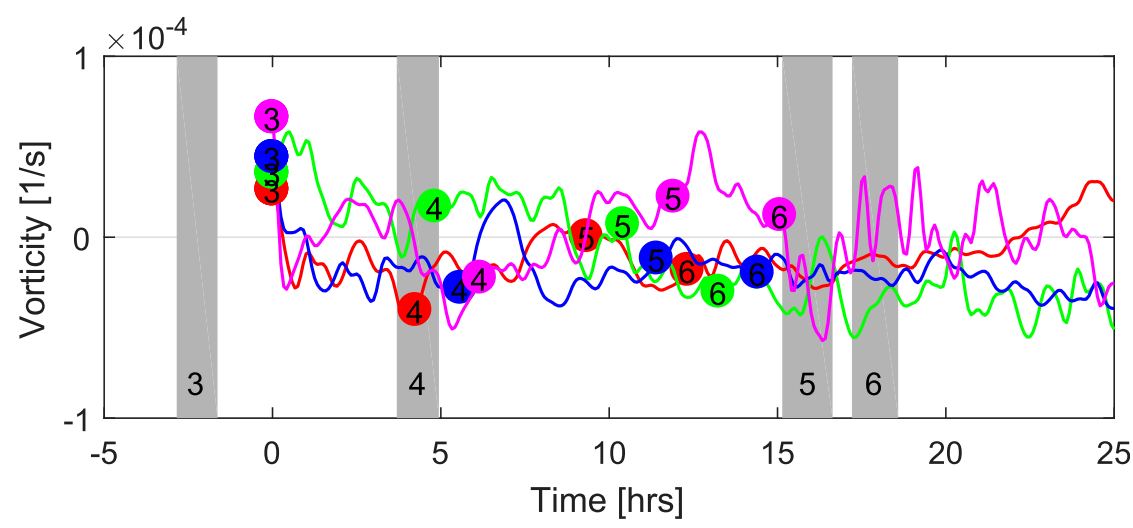

FIG. 15. Drifter-based estimate of vorticity at four depths (color coded as in Fig. 2) at the release time and at the time when the drifters are crossing sections \#4, \#5, and \#6. Shaded regions correspond to the time interval when the section was taken. 
drifter-based and Eulerian model divergence and vorticity (interpolated from the model grid to the center of mass of the drifter distribution in each layer) at all depths. The only exception occurs within a period starting at around hour 15 and at 1-m depth, where the drifters form a highly elongated distribution, leading to the unreliable estimates of velocity gradients and causing large deviations of the drifter-based divergence and vorticity from the model truth. Correlation between the simulated Lagrangian drifter-based and the Eulerian model vertical velocity is good at $1 \mathrm{~m}$ but poor at all three deeper levels $(10,30$, and $50 \mathrm{~m})$. Additional model simulations suggest that the errors in vertical velocity are, for the most part, due to the lateral separation between drifters at different depths. The second, smaller but still not insignificant, source of errors for $w$ comes from having only four vertical layers, whereas errors due to the use of drifter-derived Lagrangian divergence instead of Eulerian model divergence when estimating $w$ are less important. Overall, and despite these sources of uncertainties, this study demonstrates that the analysis of multilevel drifters provides very valuable information about the $3 \mathrm{D}$ ocean flow structure, as long as the lateral separation distance between drifters remains small.

Despite the very high spatiotemporal resolution $(400 \mathrm{~m}$ and 2 min) of our ocean circulation model, the magnitudes of divergence, vorticity, and $w$ in the model are still smaller by a factor of 2-3 than those based on real drifters. It remains to be seen in a future study whether increasing the model resolution beyond $400 \mathrm{~m}$, as well as including the nonhydrostatic effects, will bring the simulated divergence, vorticity, and $w$ values all the way up to the values inferred from real drifters.

The subduction and upwelling processes, and the associated vertical velocities, are difficult to measure in the real ocean. While ADCP technology is well established for obtaining the horizontal velocity measurements, it lacks the precision needed for reliably measuring the vertical velocities. One instrument that can directly measure the 3D movement of water in the ocean is the 3D Lagrangian float (D'Asaro 2003; D'Asaro et al. 2018) —a nontrivial oceanographic instrument in both its construction and usability. Analysis of the 3D Lagrangian float data, as well as the analysis of other drifters (SVP, CODE, and CARTHE) deployed during the 2018 CALYPSO cruise can be found in Tarry et al. (2021). Vertical velocities can also be inferred from the gradients of horizontal velocity by invoking the incompressibility condition (as was done in this paper using multilevel drifter data), or from the velocity and density fields by assuming a certain dynamical regime, for example, by employing the omega equation (Rudnick 1996). However, estimating vertical velocity in this manner requires availability of the spatial fields of horizontal velocity and/or density, from which spatial derivatives of both quantities need to be estimated. While such observations are possible, they usually require employing multiple observing platforms, such as two or more ships or gliders moving in parallel. In contrast, a handful of sections cutting through the domain of interest, such as the u-CTD and ADCP data presented here, are suboptimal for the derivative estimation due to both the limited number and extent of the sections, as well as the spatiotemporal aliasing. However, the CTD and ADCP data are extremely useful for understanding and interpreting the overall geometry of the flow and for putting other types of measurements, such as drifter data, into perspective. In general, investigation of the subduction/upwelling processes and their underlying dynamics greatly benefits from complementing data with a model. During the 2018 CALYPSO experiment, three gliders were collecting data for 2.5 months along three parallel lines crossing the Almeria-Oran front. Analysis of these data are currently in progress but preliminary results indicate vertical velocities on the order of $20 \mathrm{~m} \mathrm{day}^{-1}$ (N. Zarokanellos 2020, personal communication). Note, however, that the region sampled by the gliders was farther east from our drifters at the time of the drifter deployment.

With the GPS technology rapidly improving and becoming both more accurate and more affordable, multilevel drifter deployments similar to the one described in this paper, can provide an easy, efficient, and cost-effective way to explore both the horizontal and vertical transport properties of the flow throughout the top part of the water column. Improvements to the GPS horizontal positioning accuracy will lead to the improvement in the accuracy of the inferred flow properties, and improved vertical positioning accuracy might allow for the direct estimation of the vertical velocity at the ocean surface.

Our experiment involved a limited number of drifters that sampled the flow at four depths down to $50 \mathrm{~m}$. However, drifters with a similar design, but with a larger drogue and a thinner cable (to minimize the influence of the drag on the cable) connecting the drogue to the surface buoy, could easily reach as deep at $100 \mathrm{~m}$. Thus, future multilevel drifter experiments might allow observing and quantifying the 3D flow properties throughout the top $100 \mathrm{~m}$ of the water column.

This study involved drifters sampling the flow in a specific geographical region dominated by a strong jet at the periphery of a mesoscale anticyclonic gyre. Thus, our conclusions about the magnitudes and the spatial structure of the vertical velocity, vorticity, and divergence, as well as the balance of terms in the vorticity equation and the importance of Ekman currents, may only apply to the targeted flow regime. However, a similar sampling strategy involving multilevel drifter arrays is very promising and can be applied to other geographical regions with different flow features and different dynamical regimes.

Acknowledgments. Authors Rypina and Pratt were supported by U.S. Office of Naval Research (ONR) Grant N000141812417. Author Getscher acknowledges support from the U.S. Navy Civilian Institution Office with the MIT-WHOI Joint Program. Author Mourre acknowledges support from ONR Grant N00014-16-1-3130. We also thank Eugenio Cutolo for the initial technical support in the implementation of the ultra-high-resolution WMOP simulation. CALYPSO is a Departmental Research Initiative funded by the ONR. It is a collaborative program involving more than 30 scientists and students and multiple institutions in the United States, Spain, and Italy. Measurements were conducted from the NRV Alliance, Pourquoi Pas?, and SOCIB. We are grateful to the captains and crews of these research vessels and the technical and scientific staff involved in making measurements, running models, analyzing data, and providing support, and we thank Ben Hodges for his help with the multilevel drifter deployment. 
Data availability statement. The multilevel drifter data and the SOCIB WMOP numerical model output, as well as the $\mathrm{u}-\mathrm{CTD}$ and ADCP data along six sections that were used in this paper are available upon request from the corresponding author I. Rypina at WHOI.

\section{REFERENCES}

Albérola, C., and Coauthors, 1995: Tidal currents in the western Mediterranean Sea. Oceanol. Acta, 18, 273-284.

Berta, M., A. Griffa, T. M. Özgökmen, and A. C. Poje, 2016: Submesoscale evolution of surface drifter triads in the Gulf of Mexico. Geophys. Res. Lett., 43, 11751-11 759, https://doi.org/ 10.1002/2016GL070357.

Chen, C., and Coauthors, 2014: Process modeling studies of physical mechanisms of the formation of an anticyclonic eddy in the central Red Sea. J. Geophys. Res. Oceans, 119, 1445-1464, https://doi.org/10.1002/2013JC009351.

D'Asaro, E. A., 2003: Performance of autonomous Lagrangian floats. J. Atmos. Oceanic Technol., 20, 896-911, https://doi.org/ 10.1175/1520-0426(2003)020<0896:POALF $>2.0 . C O ; 2$.

— , and Coauthors, 2018: Ocean convergence and the dispersion of flotsam. Proc. Natl. Acad. Sci. USA, 115, 1162-1167, https:// doi.org/10.1073/pnas.1718453115.

— standing small-scale open ocean circulation during the Gulf of Mexico Research Initiative Era. Front. Mar. Sci., 7, 349, https://doi.org/10.3389/fmars.2020.00349.

Dutton, J. A., 2002: The Ceaseless Wind: An Introduction to the Theory of Atmospheric Motion. Dover Publications, 617pp.

Efron, B., 1979: Bootstrap methods: Another look at the jackknife. Ann. Stat., 7, 1-26, https://doi.org/10.1214/aos/1176344552.

Essink, S., 2019: Lagrangian dispersion and deformation in submesoscale flows. Ph.D. thesis, Massachusetts Institute of Technology, 123 pp., https://dspace.mit.edu/handle/1721.1/122330.

Garcia-Jove, M., and Coauthors, 2020: Scale-dependency of vertical velocities in the Alboran Sea through high-resolution simulations and glider observations. Ocean Sciences Meeting 2020, San Diego, CA, Amer. Geophys. Union, Abstract PS24C-2874, https://agu.confex.com/agu/osm20/meetingapp.cgi/ Paper/653361.

Haller, G., 2002: Lagrangian coherent structures from approximate velocity data. Phys. Fluids, 14, 1851-1861, https://doi.org/ 10.1063/1.1477449.

_ 2015: Lagrangian coherent structures. Annu. Rev. Fluid Mech., 47, 137-162, https://doi.org/10.1146/annurev-fluid-010313-141322.

Haza, A. C., T. M. Özgökmen, A. Griffa, A. C. Poje, and M. P. Lelong, 2014: How does drifter position uncertainty affect ocean dispersion estimates? J. Atmos. Oceanic Technol., 31, 2809-2828, https://doi.org/10.1175/JTECH-D-14-00107.1.

Hernandez-Lasheras, J., and B. Mourre, 2018: Dense CTD survey versus glider fleet sampling: Comparing data assimilation performance in a regional ocean model west of Sardinia. Ocean Sci., 14, 1069-1084, https://doi.org/10.5194/os-14-1069-2018.

Holton, J. R., and G. J. Hakim, 2013: An Introduction to Dynamic Meteorology. Elsevier Science, 552 pp.

Hoskins, B. J., 1982: The mathematical theory of frontogenesis. Annu. Rev. Fluid Mech., 14, 131-151, https://doi.org/10.1146/ annurev.fl.14.010182.001023.

Juza, M., and Coauthors, 2016: SOCIB operational ocean forecasting system and multi-platform validation in the western Mediterranean Sea. J. Oper. Oceanogr., 9, 155-166, https:// doi.org/10.1080/1755876X.2015.1117764.
Kamenkovich, I., I. I. Rypina, and P. Berloff, 2015: Properties and origins of the anisotropic eddy-induced transport in the North Atlantic. J. Phys. Oceanogr., 45, 778-791, https://doi.org/ 10.1175/JPO-D-14-0164.1.

Kirwan, A. D., Jr., and M. S. Chang, 1979: Effect of sampling rate and random position error on analysis of drifter data. J. Phys. Oceanogr., 9, 382-387, https://doi.org/10.1175/1520-0485(1979) $009<0382$ :EOSRAR $>2.0 . \mathrm{CO} ; 2$.

Lekien, F., and S. D. Ross, 2010: The computation of finite-time Lyapunov exponents on unstructured meshes and for nonEuclidean manifolds. Chaos, 20, 017505, https://doi.org/10.1063/ 1.3278516 .

Molinari, R., and A. D. Kirwan, 1975: Calculations of differential kinematic properties from Lagrangian observations in the western Caribbean Sea. J. Phys. Oceanogr., 5, 483-491, https://doi.org/10.1175/1520-0485(1975)005<0483:CODKPF> 2.0.CO;2.

Mourre, B., and Coauthors, 2018: Assessment of high-resolution regional ocean prediction systems using multi-platform observations: Illustrations in the western Mediterranean Sea. New Frontiers in Operational Oceanography, CreateSpace Independent Publishing, 663-694, https://www.godae.org/ godaedata/School/Chapter24_Mourre_et_al.pdf.

Ohlmann, J. C., P. F. White, A. L. Sybrandy, and P. P. Niiler, 2005: GPS-cellular drifter technology for coastal ocean observing systems. J. Atmos. Oceanic Technol., 22, 1381-1388, https:// doi.org/10.1175/JTECH1786.1.

Pedlosky, J., 1987: Geophysical Fluid Dynamics. 2nd ed. SpringerVerlag, $710 \mathrm{pp}$.

Rudnick, D. L., 1996: Intensive surveys of the Azores Front: 2. Inferring the geostrophic and vertical velocity fields. J. Geophys. Res., 101, 16291-16303, https://doi.org/10.1029/96JC01144.

Rypina, I. I., L. J. Pratt, J. Pullen, J. Levin, and A. Gordon, 2010: Chaotic advection in an archipelago. J. Phys. Oceanogr., 40, 1988-2006, https://doi.org/10.1175/2010JPO4336.1.

—, I. Kamenkovich, L. J. Pratt, and P. Berloff, 2012: Eddyinduced particle dispersion in the North Atlantic. J. Phys. Oceanogr., 42, 2206-2228, https://doi.org/10.1175/JPO-D-11-0191.1.

_ , A. R. Kirincich, R. Limeburner, and I. A. Udovydchenkov, 2014: Eulerian and Lagrangian correspondence of highfrequency radar and surface drifter data: Effects of radar resolution and flow components. J. Atmos. Oceanic Technol., 31, 945-966, https://doi.org/10.1175/JTECH-D-13-00146.1.

_ A. Kirincich, S. Lentz, and M. Sundermeyer, 2016: Investigating the eddy diffusivity concept in the coastal ocean. J. Phys. Oceanogr., 46, 2201-2218, https://doi.org/10.1175/JPO-D-160020.1.

Spydell, M. S., F. Feddersen, and J. Macmahan, 2019: The effect of drifter GPS errors on estimates of submesoscale vorticity. J. Atmos. Oceanic Technol., 36, 2101-2119, https://doi.org/10.1175/ JTECH-D-19-0108.1.

Sulman, M. H. M., H. S. Huntley, B. L. Lipphardt Jr., and A. D. Kirwan Jr., 2013: Leaving flatland: Diagnostics for Lagrangian coherent structures in three-dimensional flows. Physica D, 258, 77-92, https://doi.org/10.1016/j.physd.2013.05.005.

Tarry, D. L. and Coauthors, 2021: Frontal convergence and vertical velocity measured by drifters in the Alboran Sea. J. Geophys. Res. Oceans, 126, e2020JC016614, https://doi.org/10.1029/ 2020JC016614.

Tintoré, J. and Coauthors, 2013: SOCIB: The Balearic Islands coastal ocean observing and forecasting system responding to science, technology and society needs. Mar. Technol. Soc. J., 47, 101-117, https://doi:10.4031/MTSJ.47.1.10. 